\title{
Distribution of Body Mass Index of Indian Women: A Study Based on NFHS-2 and NFHS-3 Ramkrishna L. Shinde ${ }^{1 *}$ and Yashwant P. Patil ${ }^{2}$ \\ ${ }^{1}$ Department of Statistics and Actuarial Science \\ School of Mathematical Sciences,North Maharashtra University Jalgaon-425001, Maharashtra, India \\ ${ }^{2}$ Department of Statistics,J.D.M.V.P. Co. Op. Samaj's Arts, Commerce and Science College, Jalgaon-425001, Maharashtra, India \\ "Corresponding Author: rlshinde@nmu.ac.in, shinde.stat@gmail.com (iD http://orcid.org/0000-0002-1320-3937
}

Received: $10^{\text {th }}$ January, 2017/ Revised: $3^{\text {rd }}$ November, 2017/ Accepted: $7^{\text {th }}$ December, 2017 CIAppStat-SL2017

\begin{abstract}
World Health Organization recommended Body Mass Index (BMI) as a measure of nutritional status of adults. This study investigates the distribution of BMI and its changes among Indian women in the age-group 15-49 years based on sampled data of 83,646 and 111,983 women from the National Family Health Survey-2 (NFHS-2) and NFHS-3 respectively. Background characteristics (BC) specific distributional changes in BMI are demonstrated by (i) fitting an appropriate probability distributions (ii) partial sum based on percentiles of distribution and (iii) test of equality of percentiles of distribution of BMI from NFHS-2 and NFHS-3. Relative measures $R_{1}$ and $R_{2}$ are defined to demonstrate burden of underweight and obesity. Changes in the prevalence of underweight, obesity and annual gain in mean BMI for synthetic cohort by age-groups are presented. Rapid increments are observed in mean and higher percentiles of BMI among married women, women in the higher age-groups, and women from south zone of India. High prevalence of underweight is observed among rural women (40.6\%) and women from low standard of living (49.7\%). Double burden of underweight and obesity is reported among older, rich and highly educated women and women from Christian and Sikh religions. Tests of equality of percentiles of BMI are rejected $(p<0.001)$ for many $B C$. Results may be useful for making health policy for Indian women. India need to address women health issues afflicting the underweight from poor class and obesity from the rich class.
\end{abstract}


KEYWORDS: $B M I, N F H S$, underweight, obesity, change in distribution, synthetic cohort.

\section{Introduction}

The Body Mass Index (BMI) or Quetelet Index is a statistical measure of the weight of a person scaled according to height. The $B M I$ of a person is the ratio of person's weight in kilograms to the square of the height in meters $\left(\mathrm{kg} / \mathrm{m}^{2}\right)$. Researchers have demonstrated the population based changes in $B M I$ through the change in mean $B M I$ or point estimates such as prevalence of obesity to summarize changes in overall distribution (Headley et al, 2004; Flegal et al., 2010; Ogden et al., 2014). The implicit assumption in most of the studies was that the distribution of $B M I$ or the dispersion in the population has remained constant, with the entire distribution moving typically to right, allowing summaries of population change by examining a single parameter. Previous research had showed greater increases in the upper deciles of the $B M I$ distribution accompanied by increases in the variance across United states for birth cohorts from 1882 to 1986 (Komlos \& Brabee, 2011). There has also been evidence of grater increases in $B M I$ at higher quantiles within socioeconomic and demographic groups (Ljungvall \& Zimmerman, 2012). The prevalence and distribution of malnutrition in a society have implications for public health outcomes and policy formulation. Underweight people die at much higher rates, perhaps because of diseases related to being malnourished, while overweight and obese people also have higher rates of death and disease, mostly from causes such as diabetes, heart disease and cancer (Razaket al., 2013). This growing trend of body weight extremes is going to pose a major challenge for health care and policy leaders (Razaket al., 2013). While exploring the prevalence of BMI categories and changes in mean $B M I$ are important for understanding population health, they do not capture the whole distribution of BMI. Understanding the actual distribution of $B M I$ is important since mean values may hide differences in patterns at both the upper and lower ends of the distribution.

Major objective of this study is to investigate the distributional changes in BMI of Indian women from NFHS-2 to NFHS-3. For this study, we have used data from NFHS-2 and NFHS-3. These nationwide surveys were conducted in India during 1998-99 and 2005-06 respectively. We have demonstrated the changes in the distribution of $B M I$ of Indian women for some selected background characteristics $(B C)$ using different approaches.

The paper is organized as follows. Methodology is described in Section 2, which include different approaches for studying change in distribution of $B M I$ and derived output Tables of analysis. The results extracted from output Tables of 
Section 2 are reported in Section 3. Discussion on results is also included in Section 3. In Section 4, we have given brief conclusion of the study.

\section{Methodology}

\subsection{Data from NFHS-2 and NFHS-3}

In India, the National Family Health Survey (NFHS) program started in the early 1990s and it provides a nationally important source of representative data on population health for Indian states. NFHS program was initiated by the Ministry of Health and Family Welfare, Government of India. So far four rounds of NFHS are conducted namely NFHS-1 (1992-93), NFHS-2 (1998-99), NFHS-3 (2005-06) and NFHS-4 (2015-16). These are nationwide surveys conducted with a representative sample of households throughout the country. In NFHS-1 and NFHS-2 data were collected on women only. Data on height and weight of women were not collected in NFHS-1. In NFHS-2, information was collected from 91,196 households and interviewed 89,199 ever married women aged 15-49 years. Women who were pregnant at the time of survey, women who gave birth during the two months preceding the survey and women for whom there was no information on height and/or weight or for whom a $B M I$ could not be estimated are excluded from the analysis. After omission cases of such women the total sample size from India during NFHS-2 became 83,646 ever married women. In NFHS-2, the response rate was $93.7 \%$ (IIPS, 2000).

In NFHS-3, for the participation 1,31,596 women aged 15-49 years were selected in the sample. Out of 1,31,596 selected women, $1,24,385$ actually participated in the survey, yielding a response rate of $94.5 \%$. After deleting the cases of not eligible women and women with missing observations on some $\mathrm{BC}$, we have used data of 1,11,983 women (IIPS, 2007) from NFHS-3. We could not extend our study on NFHS-4as data onNFHS-4 is not open in public domain till date.

For this study, $B M I$ was considered as response variable. Categorical groups based on ranges of $B M I$ are defined using the criteria established by World Health Organization and modified guidelines given by Health Ministry of India jointly with Indian Council of Medical Research (WHO, 2004). These four groups are as follows:

Underweight (UW): $B M I<18.5 \quad$ Normal weight (NW): $18.5 \leq B M I<23$

Overweight (OW): $23 \leq B M I<25$ Obese (OB): $B M I \geq 25$

In this study, we considered some socio-economic and demographic variables as $B C$ : age-group, formal educational level, religion, caste, type of residence, marital status, zone and standard of living index of the household of each 
respondent. These variables were also considered and discussed in detail by Patil and Shinde (2014).

\subsection{Methods for studying change in distribution of $B M I$}

To demonstrate the changes in the distribution of $B M I$ of Indian women based on the survey data of NFHS-2 and NFHS-3, we have used following approaches: (i)prevalence rates and descriptive statistics of $B M I$ (ii) longitudinal changes using synthetic cohorts based on age-groups (iii) fitting an appropriate probability distribution to $B M I$ (iv) partial sums based on percentiles of distribution of $B M I$ and (v) test of equality of percentiles of distribution of $B M I$ from NFHS-2 and $N F H S$-3. In the following subsections we have explained these approaches briefly and presented the tables derived from analysis.

\subsubsection{Prevalence of underweight and obesity in Indian women}

Using the categorical groups based on ranges of $B M I$, we define following two measures $R_{1}$ and $R_{2}$ to assess burden of malnutrition (UW/OW/OB) on population.

$$
\begin{aligned}
& \mathrm{R}_{1}=\frac{\text { Prevalence percentage of women under }(U W+O W+O B)}{\text { Prevalence percentage of women under } N W} \\
& \mathrm{R}_{2}=\frac{\text { Prevalencepercentage of women under }(O W+O B)}{\text { Prevalence percentage of women under } W W}
\end{aligned}
$$

Interpretations based on values of $\mathrm{R}_{1}$ and $\mathrm{R}_{2}$ are as follows.

- If $\mathrm{R}_{1}<1$ then more than $50 \%$ of population belongs to NW. This case is rare under $B C$ for Indian women (see Table 1).

- If $\mathrm{R}_{1}>1$ then more than $50 \%$ of population is under malnutrition (UW/OW/OB) status. This case is commonly observed for many $B C$.

- $\quad$ IfR $_{1}>1$ and $R_{2}>1$ then there is double burden of UW as well as obesity $(\mathrm{OW} / \mathrm{OB})$ and problem of obesity is of more concern than UW.

- If $\mathrm{R}_{1}>1$ and $\mathrm{R}_{2}<1$ then problem of $\mathrm{UW}$ is of more concern than obesity (OW/OB).

The weighted and un-weighted prevalence percentages based on ranges of $B M I$ of Indian women according to $B C$ are presented in Table 1 for NFHS-2 and $N F H S$-3. The values of $\mathrm{R}_{1}$ and $\mathrm{R}_{2}$ based on weighted prevalence percentages are also presented in Table 1. Results are discussed in Section 3.

\subsubsection{Descriptive statistics of BMI of Indian women}

The weighted descriptive statistics (Mean, Standard Deviation (SD), some 
Table 1: Weighted and Un-weighted (in parenthesis) prevalence according to $B M I$ ranges and $B C$ of Indian women during NFHS-2 and NFHS-3

\begin{tabular}{|c|c|c|c|c|c|c|c|c|}
\hline \multirow{2}{*}{$B C$} & \multirow{2}{*}{ Survey } & \multirow{2}{*}{$\begin{array}{c}\text { Sample } \\
\text { size }\end{array}$} & \multicolumn{4}{|c|}{ Prevalence in \% } & \multirow{2}{*}{$\mathbf{R}_{1}$} & \multirow{2}{*}{$\mathbf{R}_{2}$} \\
\hline & & & UW & NW & OW & $\overline{\mathbf{O B}}$ & & \\
\hline \multicolumn{9}{|l|}{ Residence } \\
\hline \multirow{2}{*}{ Rural } & NFHS-2 & 57561 & $39.7(36.1)$ & $48.3(50.6)$ & $6.2(6.9)$ & $5.8(6.4)$ & 1.07 & 0.30 \\
\hline & NFHS-3 & 61293 & $40.6(35.6)$ & $45.0(47.7)$ & $7.0(8.1)$ & $7.4(8.6)$ & 1.22 & 0.35 \\
\hline \multirow{2}{*}{ Urban } & NFHS-2 & 26085 & $22.4(20.4)$ & 41.1(41.9) & $13.4(13.7)$ & $23.1(24.1)$ & 1.43 & 1.63 \\
\hline & NFHS-3 & 50690 & $25.0(22.5)$ & $39.5(41.5)$ & $12.0(12.5)$ & $23.5(23.5)$ & 1.53 & 1.42 \\
\hline \multicolumn{9}{|l|}{ Age-group } \\
\hline \multirow{2}{*}{$15-19$} & NFHS-2 & 6368 & $38.6(36.0)$ & $55.8(57.5)$ & $3.9(4.5)$ & $1.7(2.0)$ & 0.79 & 0.15 \\
\hline & NFHS-3 & 21650 & $46.8(42.1)$ & $47.1(49.9)$ & $3.7(4.8)$ & $2.4(3.2)$ & 1.12 & 0.13 \\
\hline \multirow{2}{*}{$20-24$} & NFHS-2 & 14725 & $39.5(35.9)$ & $51.3(53.8)$ & $5.5(6.2)$ & $3.7(4.1)$ & 0.95 & 0.23 \\
\hline & NFHS-3 & 18902 & $40.8(34.9)$ & $47.0(50.5)$ & $6.4(7.9)$ & $5.8(6.7)$ & 1.13 & 0.30 \\
\hline \multirow{2}{*}{$25-29$} & NFHS-2 & 16934 & $37.9(33.6)$ & $47.4(49.8)$ & $7.4(8.4)$ & $7.3(8.2)$ & 1.11 & 0.39 \\
\hline & NFHS-3 & 17921 & $35.3(29.4)$ & $45.0(47.1)$ & $9.1(10.5)$ & $10.6(12.9)$ & 1.22 & 0.56 \\
\hline \multirow{2}{*}{$30-34$} & NFHS-2 & 14745 & $34.3(30.1)$ & $45.0(46.6)$ & $8.9(10.0)$ & $11.8(13.4)$ & 1.22 & 0.60 \\
\hline & NFHS-3 & 16384 & $32.9(26.1)$ & $41.6(43.3)$ & $10.2(12.0)$ & $15.3(18.6)$ & 1.40 & 0.78 \\
\hline \multirow{2}{*}{$35-39$} & NFHS-2 & 12791 & $31.3(27.9)$ & 43.3(44.4) & $10.5(11.2)$ & $14.9(17.1)$ & 1.31 & 0.81 \\
\hline & NFHS-3 & 15233 & $28.9(22.8)$ & $40.3(40.8)$ & $11.2(12.6)$ & $19.6(23.8)$ & 1.48 & 1.07 \\
\hline \multirow{2}{*}{$40-44$} & $\mathrm{NF}$ & 10187 & $31.4(27.6)$ & $40.7(41.7)$ & $10.6(11.1)$ & 17.3( & 1.46 & 0.89 \\
\hline & NFHS-3 & 12507 & $27.1(20.5)$ & $38.2(38.3)$ & $11.8(13.5)$ & $23.0(27.8)$ & 1.62 & 1.28 \\
\hline \multirow{2}{*}{$45-49$} & NFHS-2 & 7896 & $29.9(26.3)$ & $40.1(40.9)$ & $10.6(11.6)$ & $19.4(21.2)$ & 1.49 & 1.00 \\
\hline & NFHS-3 & 9386 & $25.6(20.1)$ & $37.2(36.2)$ & $12.5(14.1)$ & $24.7(29.6)$ & 1.69 & 1.45 \\
\hline \multicolumn{9}{|l|}{ Education } \\
\hline \multirow{2}{*}{$\begin{array}{c}\text { No } \\
\text { Education }\end{array}$} & NFHS-2 & 41048 & $42.0(38.5)$ & $47.5(49.5)$ & $5.8(6.3)$ & $4.7(5.6)$ & 1.11 & 0.25 \\
\hline & NFHS-3 & 35500 & $41.7(36.6)$ & $44.4(45.7)$ & $6.7(8.1)$ & $7.3(9.6)$ & 1.25 & 0.34 \\
\hline \multirow{2}{*}{ Primary } & NFHS-2 & 14505 & $35.2(31.5)$ & $46.2(48.3)$ & $8.2(9.1)$ & $10.5(11.2)$ & 1.17 & 0.53 \\
\hline & NFHS-3 & 16157 & $34.9(29.9)$ & $44.1(46.2)$ & $8.8(10.0)$ & $12.2(14.0)$ & 1.27 & 0.60 \\
\hline \multirow{2}{*}{ Secondary } & NFHS-2 & 20352 & $26.1(23.0)$ & $45.9(47.2)$ & $11.0(11.8)$ & $17.0(18.1)$ & 1.18 & 1.07 \\
\hline & NFHS-3 & 48932 & $32.4(27.5)$ & $42.2(44.8)$ & $9.5(10.5)$ & $15.9(17.1)$ & 1.37 & 0.78 \\
\hline \multirow{2}{*}{ Higher } & NFHS-2 & 7720 & $15.5(13.3)$ & $40.8(40.2)$ & $15.3(16.2)$ & $28.5(30.3)$ & 1.45 & 2.83 \\
\hline & NFHS-3 & 11382 & $19.3(16.6)$ & $40.0(41.1)$ & $14.1(14.7)$ & $26.7(27.6)$ & 1.50 & 2.11 \\
\hline
\end{tabular}

Table 1 continued...

\begin{tabular}{|c|c|c|c|c|c|c|c|c|}
\hline \multirow{2}{*}{$B C$} & \multirow{2}{*}{ Survey } & \multirow{2}{*}{$\begin{array}{c}\text { Sample } \\
\text { size }\end{array}$} & \multicolumn{4}{|c|}{ Prevalence in \% } & \multirow[b]{2}{*}{$\mathbf{R}_{1}$} & \multirow{2}{*}{$\mathbf{R}_{2}$} \\
\hline & & & UW & NW & OW & OB & & \\
\hline \multicolumn{9}{|c|}{ Standard of living } \\
\hline \multirow{2}{*}{ Low } & NFHS-2 & 23118 & $6.9(44.0)$ & $46.3(48.7)$ & $4.3(4.7)$ & $2.6(2.7)$ & 1.16 & 0.15 \\
\hline & NFHS-3 & 18051 & $49.7(45.5)$ & $42.8(46.0)$ & $4.4(5.2)$ & $3.0(3.4)$ & 1.33 & 0.15 \\
\hline \multirow{2}{*}{ Medium } & NFH & 39722 & $34.8(31.7)$ & $48.5(50.5)$ & $8.1(8.7)$ & $8.5(9.1)$ & 1.06 & 0.48 \\
\hline & NFH & 32272 & $40.2(35.1)$ & $45.7(48.6)$ & $6.9(8.0)$ & $7.2(8.4)$ & 1.19 & 0.35 \\
\hline \multirow{2}{*}{ High } & NFHS-2 & 19812 & $17.2(15.5)$ & $41.5(41.5)$ & $14.3(14.7)$ & $27.0(28.2)$ & 1.41 & 2.40 \\
\hline & NFH & 54740 & $24.3(21.1)$ & $41.0(42.2)$ & 12.1(12.9) & $22.5(23.8)$ & 1.44 & 1.42 \\
\hline \multicolumn{9}{|l|}{ Religion } \\
\hline \multirow{2}{*}{ Hindu } & $\mathrm{NF}$ & 65069 & $36.3(33.2)$ & $46.6(47.3)$ & $7.7(8.5)$ & $9.3(10.9)$ & 1.14 & 0.47 \\
\hline & NFHs & 81342 & $36.4(31.9)$ & $43.5(43.7)$ & $8.3(9.6)$ & $11.8(14.8)$ & 1.30 & 0.55 \\
\hline \multirow{2}{*}{ Muslim } & NFHS-2 & 9734 & $32.9(29.5)$ & $45.7(47.2)$ & $9.1(9.8)$ & $12.3(13.6)$ & 1.19 & 0.65 \\
\hline & NFHS-3 & 14452 & $35.1(30.1)$ & $42.1(42.0)$ & $8.6(10.3)$ & $14.1(17.6)$ & 1.37 & 0.65 \\
\hline \multirow{2}{*}{ Christian } & NFHS-2 & 4709 & $24.4(19.3)$ & $47.1(56.4)$ & $11.4(11.4)$ & $17.1(13.0)$ & 1.12 & 1.17 \\
\hline & & 10024 & $23.2(16.8)$ & $45.8(58.5)$ & $12.8(12.1)$ & $18.2(12.5)$ & 1.18 & 1.34 \\
\hline Sikh & NFHS-2 & 1998 & $16.3(16.4)$ & $40.7(39.9)$ & $13.8(13.6)$ & $29.2(30.1)$ & 1.46 & 2.64 \\
\hline
\end{tabular}




\begin{tabular}{|c|c|c|c|c|c|c|c|c|}
\hline & NFHS-3 & 2591 & $17.8(17.9)$ & $36.7(36.6)$ & $13.9(13.7)$ & $31.6(31.8)$ & 1.72 & 2.56 \\
\hline \multirow{2}{*}{ Other } & NFHS-2 & 2136 & $23.6(17.6)$ & $43.2(56.1)$ & $9.3(11.8)$ & $14.8(14.5)$ & 1.10 & 1.02 \\
\hline & NFHS-3 & 3574 & $36.8(21.7)$ & $42.4(51.1)$ & $8.8(12.7)$ & $11.9(14.5)$ & 1.36 & 0.56 \\
\hline \multicolumn{9}{|l|}{ Caste } \\
\hline \multirow{2}{*}{$\mathrm{SC}$} & NFHS-2 & 14225 & $41.2(38.7)$ & $46.9(47.5)$ & $6.1(6.8)$ & $5.7(7.0)$ & 1.13 & 0.29 \\
\hline & NFHS-3 & 18343 & $41.1(36.4)$ & $42.9(43.3)$ & $7.1(8.5)$ & $8.9(11.7)$ & 1.33 & 0.39 \\
\hline \multirow{2}{*}{ ST } & NFHS-2 & 10234 & $44.9(32.7)$ & $47.3(55.7)$ & $4.6(7.0)$ & $3.1(4.6)$ & 1.11 & 0.17 \\
\hline & NFHS-3 & 14985 & $46.6(28.3)$ & $45.2(56.3)$ & $4.7(9.1)$ & $3.5(6.3)$ & 1.21 & 0.18 \\
\hline \multirow{2}{*}{$\mathrm{OBC}$} & NFHS-2 & 24409 & $35.0(33.2)$ & $47.8(48.3)$ & $7.9(8.5)$ & $9.3(9.9)$ & 1.09 & 0.49 \\
\hline & NFHS-3 & 35172 & $35.7(32.3)$ & $44.2(44.2)$ & $8.4(9.5)$ & $11.6(14.0)$ & 1.26 & 0.56 \\
\hline \multirow{2}{*}{$\begin{array}{c}\text { Gen and } \\
\text { Other }\end{array}$} & NFHS-2 & 34302 & $30.0(26.0)$ & $44.7(45.4)$ & $10.1(11.0)$ & $15.2(17.6)$ & 1.24 & 0.84 \\
\hline & NFHS-3 & 43483 & $29.7(25.1)$ & $41.7(42.2)$ & $10.5(11.6)$ & $18.1(21.1)$ & 1.40 & 0.96 \\
\hline \multicolumn{9}{|c|}{ Marital status } \\
\hline \multirow{2}{*}{ Married } & NFHS-2 & 78638 & $34.9(31.0)$ & $46.6(48.0)$ & $8.2(9.1)$ & $10.3(11.9)$ & 1.15 & 0.53 \\
\hline & NFHS-3 & 77524 & $33.0(26.9)$ & $42.6(43.0)$ & $9.5(11.2)$ & $14.9(18.9)$ & 1.35 & 0.74 \\
\hline \multirow{2}{*}{$\begin{array}{l}\text { Unmarried } \\
\text { and Other }\end{array}$} & NFHS-2 & 5008 & $39.2(34.7)$ & $42.9(45.6)$ & $7.7(8.4)$ & $10.2(11.2)$ & 1.33 & 0.46 \\
\hline & NFHS-3 & 34459 & $42.8(35.9)$ & $44.9(49.1)$ & $6.0(7.6)$ & $6.3(7.4)$ & 1.23 & 0.29 \\
\hline \multicolumn{9}{|l|}{ Zone } \\
\hline \multirow{2}{*}{ North } & NFHS-2 & 20750 & $26.9(24.5)$ & $47.6(44.6)$ & $9.8(9.8)$ & $15.7(21.1)$ & 1.10 & 0.95 \\
\hline & NFHS-3 & 20671 & $29.3(26.6)$ & $44.3(44.4)$ & $10.0(10.7)$ & $16.5(18.3)$ & 1.26 & 0.90 \\
\hline \multirow{2}{*}{ Central } & NFHS-2 & 16233 & $35.9(28.3)$ & $50.4(40.9)$ & $7.0(5.5)$ & $6.7(25.3)$ & 0.98 & 0.38 \\
\hline & NFHS-3 & 19850 & $38.3(35.5)$ & $46.3(44.6)$ & $7.0(7.8)$ & $8.4(12.1)$ & 1.16 & 0.40 \\
\hline \multirow{2}{*}{ East } & NFHS-2 & 15857 & $41.8(38.5)$ & $47.1(45.0)$ & $5.6(5.8)$ & $5.5(10.7)$ & 1.12 & 0.27 \\
\hline & NFHS-3 & 16441 & $41.9(37.2)$ & $43.5(43.2)$ & $7.0(8.5)$ & $7.6(11.1)$ & 1.30 & 0.35 \\
\hline \multirow{2}{*}{ North-East } & NFHS-2 & 11015 & $25.9(19.9)$ & $60.7(57.1)$ & $8.1(9.5)$ & $5.2(13.4)$ & 0.65 & 0.51 \\
\hline & NFHS-3 & 19855 & $31.9(20.2)$ & $51.3(57.5)$ & $8.8(11.7)$ & $8.0(10.6)$ & 0.95 & 0.53 \\
\hline \multirow{2}{*}{ West } & NFHS-2 & 10482 & $38.2(32.0)$ & $40.4(37.8)$ & $8.8(9.8)$ & $12.7(20.4)$ & 1.48 & 0.56 \\
\hline & NFHS-3 & 14481 & $36.2(32.9)$ & $39.1(38.9)$ & $9.4(10.6)$ & $15.3(17.6)$ & 1.56 & 0.68 \\
\hline \multirow{2}{*}{ South } & NFHS-2 & 15966 & $32.2(29.8)$ & $43.7(42.3)$ & $10.0(10.3)$ & $14.1(17.6)$ & 1.29 & 0.75 \\
\hline & NFHS-3 & 20685 & $30.6(27.8)$ & $40.4(39.1)$ & $10.4(11.0)$ & $18.6(22.0)$ & 1.48 & 0.95 \\
\hline \multirow{2}{*}{ Total } & NFHS-2 & 83646 & 35.2(28.9) & 46.4(44.3) & $8.1(8.4)$ & $10.3(18.4)$ & 1.16 & 0.52 \\
\hline & NFHS-3 & 111983 & $35.6(29.6)$ & $43.2(44.9)$ & $8.6(10.1)$ & $12.6(15.4)$ & 1.31 & 0.60 \\
\hline
\end{tabular}

SC: Scheduled Caste, ST: Scheduled Tribe, OBC: Other Backward Class percentiles, Coefficient of Skewness $\left(\beta_{1}\right)$ and $\left.\operatorname{Kurtosis}\left(\beta_{2}\right)\right)$ of BMI for women according to various $B C$ are presented in Table 2 for both surveys. Estimated annual percent gain in mean BMI during the period of NFHS-2 and NFHS-3 (in 7 years span) is calculated by using following formula.

$$
\text { Annual percent gain }=\left\{\left(\frac{\text { Mean } B M I \text { in } N F H S 3}{\text { Mean } B M I \text { in } N F H S 2}\right)^{1 / 7}-1\right\} \times 100
$$

Table 2: Weighted descriptive Statistics of BMI

\begin{tabular}{|c|c|c|c|c|c|c|c|c|c|c|c|}
\hline \multirow[b]{2}{*}{$B C$} & \multirow[b]{2}{*}{ Survey } & \multirow[b]{2}{*}{ Mean } & \multirow[b]{2}{*}{ SD } & \multicolumn{5}{|c|}{ Percentiles } & \multirow[b]{2}{*}{$\beta_{1}$} & \multirow[b]{2}{*}{$\boldsymbol{\beta}_{2}$} & \multirow{2}{*}{$\begin{array}{l}\text { Annual } \\
\% \\
\text { gainin } \\
\text { Mean } \\
\text { BMI }\end{array}$} \\
\hline & & & & 5th & 25th & 50th & 75th & 95th & & & \\
\hline \multicolumn{12}{|c|}{ Residence } \\
\hline \multirow{2}{*}{ Urban } & NFHS-2 & 22.07 & 4.48 & 16.13 & 18.78 & 21.34 & 24.69 & 30.36 & 0.90 & 1.18 & \multirow{2}{*}{-0.07} \\
\hline & NFHS-3 & 21.96 & 4.63 & 15.92 & 18.49 & 21.13 & 24.70 & 30.60 & 0.89 & 0.91 & \\
\hline Rural & NFHS-2 & 19.63 & 3.11 & 15.63 & 17.56 & 19.14 & 21.12 & 25.39 & 1.44 & 5.04 & 0.09 \\
\hline
\end{tabular}


Distribution of Body Mass Index of Indian Women

\begin{tabular}{|c|c|c|c|c|c|c|c|c|c|c|c|}
\hline & NFHS-3 & 19.75 & 3.32 & 15.61 & 17.51 & 19.12 & 21.31 & 26.17 & 1.38 & 3.82 & \\
\hline \multicolumn{12}{|l|}{ Age-group } \\
\hline \multirow{2}{*}{$15-19$} & NFHS-2 & 19.29 & 2.31 & 15.99 & 17.70 & 19.10 & 20.65 & 23.18 & 0.96 & 4.09 & \multirow{2}{*}{-0.25} \\
\hline & NFHS-3 & 18.96 & 2.54 & 15.48 & 17.28 & 18.67 & 20.27 & 23.38 & 1.35 & 8.15 & \\
\hline \multirow{2}{*}{$20-24$} & NFHS-2 & 19.46 & 2.75 & 15.80 & 17.64 & 19.11 & 20.86 & 24.31 & 1.53 & 8.99 & \multirow{2}{*}{0.12} \\
\hline & NFHS-3 & 19.62 & 3.09 & 15.72 & 17.54 & 19.08 & 21.06 & 25.45 & 1.43 & 4.28 & \\
\hline \multirow{2}{*}{$25-29$} & NFHS-2 & 19.88 & 3.28 & 15.72 & 17.67 & 19.28 & 21.47 & 26.09 & 1.33 & 3.44 & \multirow{2}{*}{0.31} \\
\hline & NFHS-3 & 20.32 & 3.66 & 15.76 & 17.76 & 19.56 & 22.17 & 27.43 & 1.21 & 2.24 & \\
\hline \multirow{2}{*}{$30-34$} & NFHS-2 & 20.47 & 3.82 & 15.76 & 17.84 & 19.73 & 22.32 & 27.80 & 1.30 & 2.83 & \multirow{2}{*}{0.27} \\
\hline & NFHS-3 & 20.86 & 4.06 & 15.80 & 17.91 & 20.02 & 23.06 & 28.65 & 1.11 & 1.99 & \\
\hline \multirow[b]{2}{*}{$35-39$} & NFHS-2 & 20.89 & 4.13 & 15.67 & 17.97 & 20.04 & 23.05 & 28.81 & 1.19 & 2.29 & \multirow{2}{*}{0.34} \\
\hline & NFHS-3 & 21.39 & 4.38 & 15.81 & 18.14 & 20.51 & 23.95 & 29.75 & 0.94 & 1.02 & \\
\hline \multirow[b]{2}{*}{$40-44$} & NFHS-2 & 21.09 & 4.33 & 15.61 & 17.94 & 20.26 & 23.51 & 29.15 & 1.09 & 1.82 & \multirow{2}{*}{0.49} \\
\hline & NFHS-3 & 21.83 & 4.67 & 15.86 & 18.28 & 20.97 & 24.59 & 30.69 & 0.90 & 0.90 & \\
\hline \multirow{2}{*}{$45-49$} & NFHS-2 & 21.31 & 4.51 & 15.48 & 18.02 & 20.50 & 23.83 & 29.96 & 0.94 & 1.08 & \multirow{2}{*}{0.50} \\
\hline & NFHS-3 & 22.07 & 4.78 & 15.81 & 18.42 & 21.24 & 24.95 & 30.88 & 0.89 & 1.00 & \\
\hline \multicolumn{12}{|l|}{ Education } \\
\hline \multirow{2}{*}{$\begin{array}{c}\text { No } \\
\text { Education } \\
\end{array}$} & NFHS-2 & 19.44 & 3.00 & 15.52 & 17.46 & 18.99 & 20.90 & 24.84 & 1.42 & 4.79 & \multirow{2}{*}{0.18} \\
\hline & NFHS-3 & 19.69 & 3.34 & 15.55 & 17.45 & 19.04 & 21.24 & 26.16 & 1.39 & 3.62 & \\
\hline \multirow{2}{*}{ Primary } & NFHS-2 & 20.30 & 3.68 & 15.74 & 17.76 & 19.61 & 22.02 & 27.41 & 1.34 & 3.15 & \multirow{2}{*}{0.12} \\
\hline & NFHS-3 & 20.47 & 3.88 & 15.74 & 17.79 & 19.66 & 22.34 & 28.03 & 1.33 & 3.30 & \\
\hline \multirow{2}{*}{ Secondary } & NFHS-2 & 21.29 & 4.10 & 16.10 & 18.40 & 20.48 & 23.46 & 29.16 & 1.14 & 2.29 & \multirow{2}{*}{-0.26} \\
\hline & NFHS-3 & 20.91 & 4.21 & 15.78 & 17.93 & 19.94 & 23.06 & 29.18 & 1.19 & 1.92 & \\
\hline \multirow{2}{*}{ Higher } & NFHS-2 & 22.89 & 4.49 & 16.72 & 19.64 & 22.25 & 25.53 & 31.05 & 0.89 & 1.53 & \multirow{2}{*}{-0.25} \\
\hline & NFHS-3 & 22.50 & 4.48 & 16.40 & 19.12 & 21.87 & 25.22 & 30.50 & 0.78 & 0.85 & \\
\hline \multicolumn{12}{|c|}{ Standard of living } \\
\hline \multirow{2}{*}{ Low } & NFHS-2 & 19.00 & 2.67 & 15.37 & 17.23 & 18.69 & 20.40 & 23.60 & 1.34 & 5.64 & \multirow[b]{2}{*}{-0.04} \\
\hline & NFHS-3 & 18.95 & 2.74 & 15.36 & 17.15 & 18.52 & 20.29 & 23.83 & 1.54 & 7.46 & \\
\hline Medium & NFHS-2 & 20.13 & 3.40 & 15.76 & 17.81 & 19.54 & 21.77 & 26.50 & 1.33 & 3.85 & \\
\hline Medıum & NFHS-3 & 19.73 & 3.27 & 15.58 & 17.51 & 19.14 & 21.34 & 26.02 & 1.26 & 3.26 & -0. \\
\hline & NFHS-2 & 22.67 & 4.49 & 16.68 & 19.34 & 21.98 & 25.31 & 31.00 & 0.88 & 1.24 & \\
\hline High & NFHS-3 & 21.90 & 4.49 & 16.16 & 18.56 & 21.05 & 24.53 & 30.36 & 0.93 & 1.04 & -0.49 \\
\hline
\end{tabular}

\begin{tabular}{|c|c|c|c|c|c|c|c|c|c|c|c|}
\hline \multicolumn{12}{|l|}{ eligion } \\
\hline \multirow{2}{*}{ Hindu } & NFHS-2 & 20.13 & 3.58 & 15.70 & 17.72 & 19.44 & 21.74 & 27.01 & 1.42 & 3.81 & \multirow{2}{*}{0.16} \\
\hline & NFHS-3 & 20.36 & 3.85 & 15.67 & 17.70 & 19.50 & 22.19 & 27.87 & 1.32 & 2.74 & \\
\hline \multirow{2}{*}{ Muslim } & NFHS-2 & 20.58 & 3.89 & 15.68 & 17.90 & 19.79 & 22.44 & 28.13 & 1.31 & 2.48 & \multirow{2}{*}{0.02} \\
\hline & NFHS-3 & 20.62 & 4.11 & 15.74 & 17.74 & 19.64 & 22.57 & 28.72 & 1.26 & 2.05 & \\
\hline \multirow{2}{*}{$\begin{array}{c}\text { Christia } \\
n\end{array}$} & NFHS-2 & 21.37 & 3.95 & 16.23 & 18.56 & 20.73 & 23.40 & 28.77 & 0.98 & 1.46 & \multirow{2}{*}{0.14} \\
\hline & NFHS-3 & 21.58 & 4.07 & 16.23 & 18.68 & 20.94 & 23.77 & 29.30 & 0.96 & 1.68 & \\
\hline \multirow{2}{*}{ Sikh } & NFHS-2 & 22.87 & 4.63 & 16.62 & 19.42 & 22.19 & 25.53 & 31.38 & 0.85 & 0.88 & \multirow{2}{*}{0.19} \\
\hline & NFHS-3 & 23.18 & 5.02 & 16.65 & 19.38 & 22.40 & 26.23 & 32.31 & 0.91 & 1.26 & \\
\hline \multirow{2}{*}{$\begin{array}{c}\text { Gen and } \\
\text { Other }\end{array}$} & NFHS-2 & 20.79 & 4.15 & 15.63 & 17.77 & 19.86 & 22.82 & 28.95 & 1.27 & 2.82 & \multirow{2}{*}{-0.32} \\
\hline & NFHS-3 & 20.31 & 3.80 & 15.60 & 17.63 & 19.49 & 22.29 & 27.63 & 1.17 & 1.95 & \\
\hline \multicolumn{12}{|l|}{ Caste } \\
\hline \multirow{2}{*}{ ST } & NFHS-2 & 19.16 & 2.75 & 15.52 & 17.33 & 18.80 & 20.55 & 23.92 & 1.43 & 5.40 & \multirow{2}{*}{-0.02} \\
\hline & NFHS-3 & 19.12 & 2.84 & 15.43 & 17.24 & 18.70 & 20.48 & 24.20 & 1.80 & 11.7 & \\
\hline \multirow{2}{*}{$\mathrm{SC}$} & NFHS-2 & 19.57 & 3.14 & 15.57 & 17.48 & 19.06 & 21.10 & 25.37 & 1.41 & 4.48 & \multirow{2}{*}{0.21} \\
\hline & NFHS-3 & 19.87 & 3.53 & 15.51 & 17.46 & 19.15 & 21.53 & 26.71 & 1.30 & 2.70 & \\
\hline \multirow{2}{*}{$\mathrm{OBC}$} & NFHS-2 & 20.18 & 3.53 & 15.76 & 17.80 & 19.55 & 21.82 & 26.86 & 1.42 & 4.42 & \multirow{2}{*}{0.12} \\
\hline & NFHS-3 & 20.36 & 3.80 & 15.70 & 17.74 & 19.53 & 22.16 & 27.79 & 1.30 & 2.64 & \\
\hline \multirow{2}{*}{ Other } & NFHS-2 & 20.97 & 4.10 & 15.86 & 18.09 & 20.12 & 23.05 & 28.92 & 1.19 & 2.11 & \multirow{2}{*}{0.19} \\
\hline & NFHS-3 & 21.26 & 4.35 & 15.88 & 18.12 & 20.31 & 23.60 & 29.66 & 1.11 & 1.61 & \\
\hline
\end{tabular}




\begin{tabular}{|c|c|c|c|c|c|c|c|c|c|c|c|}
\hline \multicolumn{12}{|c|}{\begin{tabular}{|l|} 
Marital status \\
\end{tabular}} \\
\hline \multirow{2}{*}{ Mar } & NFHS-2 & 20.29 & 3.68 & 15.74 & 17.79 & 19.58 & 21.96 & 27.38 & 1.38 & 3.45 & \multirow{2}{*}{0.36} \\
\hline & NFHS-3 & 20.81 & 4.10 & 15.81 & 17.91 & 19.88 & 22.90 & 28.79 & 1.21 & 2.14 & \\
\hline \multirow{2}{*}{ Other } & NFHS-2 & 20.08 & 3.80 & 15.41 & 17.55 & 19.29 & 21.91 & 27.40 & 1.44 & 3.80 & \multirow{2}{*}{-0.38} \\
\hline & NFHS-3 & 19.54 & 3.27 & 15.46 & 17.37 & 18.97 & 20.98 & 25.70 & 1.47 & 4.23 & \\
\hline \multicolumn{12}{|l|}{ Zone } \\
\hline \multirow{2}{*}{ North } & NFHS-2 & 21.18 & 4.12 & 16.23 & 18.36 & 20.29 & 23.08 & 29.18 & 1.35 & 2.74 & \multirow{2}{*}{-0.02} \\
\hline & NFHS-3 & 21.14 & 4.24 & 16.02 & 18.18 & 20.20 & 23.25 & 29.43 & 1.30 & 2.91 & \\
\hline \multirow{2}{*}{ Central } & NFHS-2 & 19.94 & 3.21 & 15.91 & 17.79 & 19.40 & 21.36 & 25.91 & 1.50 & 4.38 & \multirow{2}{*}{0} \\
\hline & NFHS-3 & 19.94 & 3.42 & 15.76 & 17.65 & 19.25 & 21.42 & 26.60 & 1.41 & 3.27 & \\
\hline \multirow{2}{*}{ East } & NFHS-2 & 19.48 & 3.07 & 15.52 & 17.48 & 18.99 & 20.89 & 25.31 & 1.47 & 5.55 & \multirow{2}{*}{0.18} \\
\hline & NFHS-3 & 19.73 & 3.37 & 15.55 & 17.45 & 19.04 & 21.29 & 26.26 & 1.39 & 3.85 & \\
\hline \multirow{2}{*}{$\begin{array}{c}\text { North- } \\
\text { East }\end{array}$} & NFHS-2 & 20.24 & 2.90 & 16.21 & 18.41 & 19.97 & 21.60 & 25.09 & 1.47 & 6.98 & \multirow[b]{2}{*}{0} \\
\hline & NFHS-3 & 20.24 & 3.20 & 15.98 & 18.01 & 19.73 & 21.93 & 26.29 & 1.02 & 1.85 & \\
\hline \multirow{2}{*}{ West } & NFHS-2 & 20.39 & 4.12 & 15.43 & 17.47 & 19.46 & 22.44 & 28.36 & 1.36 & 3.07 & \multirow{2}{*}{0.22} \\
\hline & NFHS-3 & 20.71 & 4.29 & 15.52 & 17.63 & 19.68 & 22.96 & 29.19 & 1.15 & 1.57 & \\
\hline \multirow{2}{*}{ South } & NFHS-2 & 20.75 & 3.95 & 15.74 & 17.91 & 19.99 & 22.86 & 28.28 & 1.09 & 1.84 & \multirow{2}{*}{0.33} \\
\hline & NFHS-3 & 21.24 & 4.39 & 15.72 & 17.99 & 20.35 & 23.66 & 29.61 & 1.03 & 1.32 & \\
\hline \multirow{2}{*}{ Total } & NFHS-2 & 20.28 & 3.68 & 15.71 & 17.78 & 19.56 & 21.96 & $\mathbf{2 7 . 3 9}$ & 1.39 & 3.48 & \multirow{2}{*}{0.13} \\
\hline & NFHS-3 & 20.47 & 3.93 & 15.69 & 17.74 & 19.59 & 22.37 & 28.18 & 1.29 & 2.38 & \\
\hline
\end{tabular}

\subsubsection{Synthetic cohort approach}

We use synthetic cohort approach to examine longitudinal change in BMI between NFHS-2 and NFHS-3, by matching the age-group level representatives by their birth year. This technique has been widely used by econometricians and involves the creation of a 'pseudo panel' from a time series of independent surveys conducted using the same methodology and on the same reference population (Lean et al., 2013 and Robinson et al., 2013). This approach is also useful in epidemiological studies of under nutrition and obesity population. In this study, we have created synthetic cohorts to determine changes in mean $B M I$ for same age-groups between 1998-99 (NFHS-2) and 2005-06 (NFHS-3). For the analysis, age-groups in six-year bands were used to facilitate comparisons between the different time periods. Five cohorts were defined for women, the youngest age-group was 15-21 years, and the oldest age-group was 36-42 years in NFHS-2 (1998-99). These cohorts were matched with the equivalent cohorts underNFHS-3(2005-06). That is, the cohort of women of age-group 15-21 years from $N F H S$-2 is matched with women aged 22-28 years from $N F H S$-3, age-group 22-28 years from NFHS-2 is matched with women aged 29-35 years from NFHS3 and so on. Thus, we can estimate longitudinal change in BMI for age stratified groups. Mean BMI within period are determined for each synthetic cohort. Finally, in order to investigate age period effect in annual $B M I$ change, change in mean $B M I$ over seven years is annualize by assuming constant annual change within period by using equation (3). The results of change in prevalence of $B M I$ 
categories and annual gain in mean $B M I$ for synthetic cohort by age-groups are reported in Table 3 and Table 4 respectively.

Table 3: Change in prevalence percentage using synthetic cohort approach

\begin{tabular}{|c|c|c|c|c|c|c|c|c|c|c|}
\hline \multirow{3}{*}{$\begin{array}{c}\text { Group } \\
\text { No. }\end{array}$} & \multirow{3}{*}{ Survey } & \multirow{3}{*}{$\begin{array}{l}\text { Age- } \\
\text { group }\end{array}$} & \multicolumn{8}{|c|}{ Weighted prevalence in \% with change } \\
\hline & & & \multicolumn{2}{|c|}{$\mathbf{U W}$} & \multicolumn{2}{|c|}{ NW } & \multicolumn{2}{|c|}{ OW } & \multicolumn{2}{|c|}{$\mathbf{O B}$} \\
\hline & & & $\%$ & Change & $\%$ & Change & $\%$ & Change & $\%$ & Change \\
\hline \multirow{2}{*}{1} & NFHS-2 & $15-21$ & 39.0 & \multirow{2}{*}{-3.84} & 54.4 & \multirow{2}{*}{-15.9} & 4.4 & \multirow{2}{*}{86.4} & 2.2 & \multirow{2}{*}{290.9} \\
\hline & NFHS-3 & $22-28$ & 37.5 & & 45.7 & & 8.2 & & 8.6 & \\
\hline \multirow{2}{*}{2} & NFHS-2 & $22-28$ & 38.7 & \multirow{2}{*}{-16.8} & 48.6 & \multirow{2}{*}{-13.6} & 6.7 & \multirow{2}{*}{53.7} & 5.9 & \multirow{2}{*}{162.7} \\
\hline & NFHS-3 & $29-35$ & 32.2 & & 42.0 & & 10.3 & & 15.5 & \\
\hline \multirow{2}{*}{3} & NFHS-2 & $29-35$ & 34.4 & \multirow{2}{*}{-18.0} & 45.2 & \multirow{2}{*}{-13.5} & 9.0 & \multirow{2}{*}{27.8} & 11.4 & \multirow{2}{*}{85.1} \\
\hline & NFHS-3 & $36-42$ & 28.2 & & 39.1 & & 11.5 & & 21.1 & \\
\hline \multirow{2}{*}{4} & NFHS-2 & $36-42$ & 31.0 & \multirow{2}{*}{-17.1} & 42.4 & \multirow{2}{*}{-11.8} & 10.3 & \multirow{2}{*}{17.5} & 16.2 & \multirow{2}{*}{53.1} \\
\hline & NFHS-3 & $43-49$ & 25.7 & & 37.4 & & 12.1 & & 24.8 & \\
\hline \multirow{2}{*}{5} & NFHS-2 & $15-42$ & 35.9 & \multirow{2}{*}{-11.1} & 47.4 & \multirow{2}{*}{-12.0} & 7.7 & \multirow{2}{*}{32.5} & 9.0 & \multirow{2}{*}{80} \\
\hline & NFHS-3 & $22-49$ & 31.9 & & 41.7 & & 10.2 & & 16.2 & \\
\hline
\end{tabular}

Table 4: Mean BMIand annual percentage gain in mean BMI for synthetic cohorts

\begin{tabular}{|c|c|c|c|c|}
\hline \multirow{2}{*}{$\begin{array}{c}\text { Age-group and } \\
\text { mean BMI in } \\
\text { NFHS-2 }\end{array}$} & \multicolumn{2}{|c|}{$\begin{array}{c}\text { Age-group and mean } \\
\text { BMI in NFHS-3 }\end{array}$} & \multicolumn{2}{c|}{$\begin{array}{c}\text { Annual gain in mean BMI } \\
\text { from NFHS-2 to NFHS-3 }\end{array}$} \\
\cline { 2 - 5 } age-group & $\begin{array}{c}\text { Came } \\
\text { age- group }\end{array}$ & $\begin{array}{c}\text { Same } \\
\text { age-group }\end{array}$ & $\begin{array}{c}\text { Cohort } \\
\text { age-group }\end{array}$ \\
\hline $\begin{array}{c}15-21 \\
(19.32)\end{array}$ & $\begin{array}{c}15-21 \\
(19.07)\end{array}$ & $\begin{array}{c}22-28 \\
(20.04)\end{array}$ & -0.18 & 0.52 \\
\hline $\begin{array}{c}22-28 \\
(19.72)\end{array}$ & $\begin{array}{c}22-28 \\
(20.04)\end{array}$ & $\begin{array}{c}29-35 \\
(20.89)\end{array}$ & 0.23 & 0.82 \\
\hline $29-35$ & $\begin{array}{c}29-35 \\
(20.89)\end{array}$ & $\begin{array}{c}36-42 \\
(21.59)\end{array}$ & 0.31 & 0.78 \\
\hline$(20.44)$ & $\begin{array}{c}36-42 \\
(21.59)\end{array}$ & $\begin{array}{c}43-49 \\
(22.05)\end{array}$ & 0.39 & 0.70 \\
\hline$(21.00)$ & $\begin{array}{c}15-42 \\
(20.47)\end{array}$ & $\begin{array}{c}22-49 \\
(20.98)\end{array}$ & 0.24 & 0.60 \\
\hline$(20.12)$ & \multicolumn{3}{|c|}{} \\
\hline
\end{tabular}




\subsubsection{Fitting of probability distribution to BMI}

For many years, prior to year 2000, the distribution of $B M I$ was assumed to follow a normal distribution or approximately a bell-shaped curve as this was similar case of measurements of weight and height. Penman and Johnson (2006) has made claim that normal distribution is not appropriate for $B M I$ indicating that some positively skewed distribution may be a better fit. Hence, the distributions, exhibiting a positive degree of skewing in the right tail of the data, were fitted using one of the parameter estimation methods namely method of moments, maximum likelihood, least square, and L-moments by Easy-Fit software. The fitted distributions of $B M I$ for various $B C$ and their estimated parameters using Easy-Fit Software (Easy-Fit) are presented in Table 5 for NFHS-2 and NFHS-3.

We observed that commonly best fitted probability distributions of $B M I$ of Indian women under different $B C$ are: Beta, Burr (4P), Dagum (3P or 4P), Fatigue Life (3P), Gamma (3P), General Gamma (4P), General extreme value, Gumbel Max, Inverse Gaussian (3P), Johnson SB, Johnson SU, and Pearson 6 (4P). These best fitted distributions and their estimated parameters are used to compare changes in the distributions of $B M I$ under different age-groups are presented in Figure 1. Probability density functions $(p d f s)$ along with parameter description of these distributions are given in Appendix.

Age Group 15-19

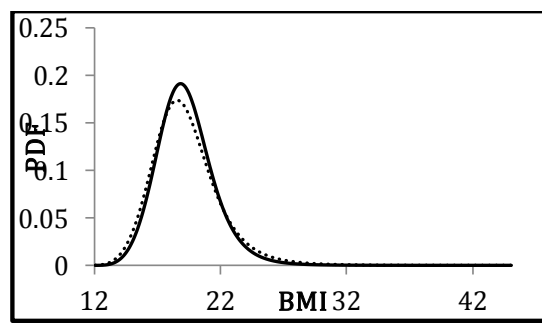

Age Group 25-29

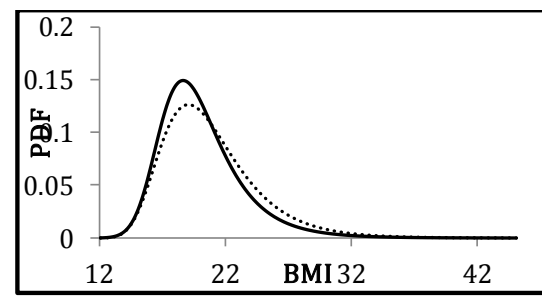

Age Group 20-24

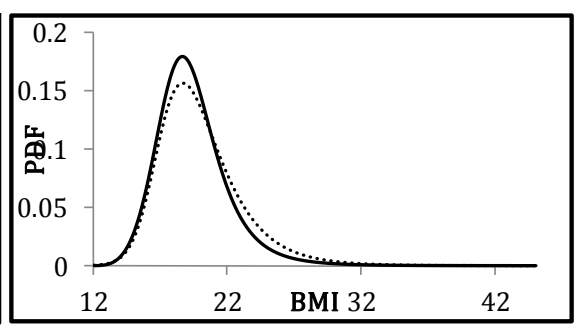

Age Group 30-34

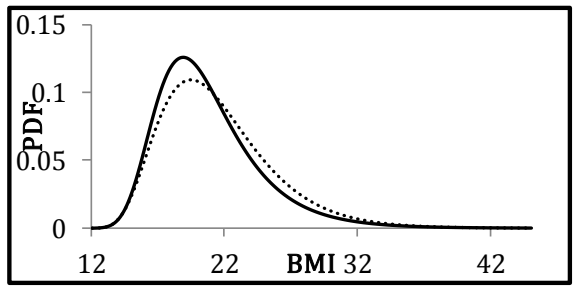


Age Group 35-39

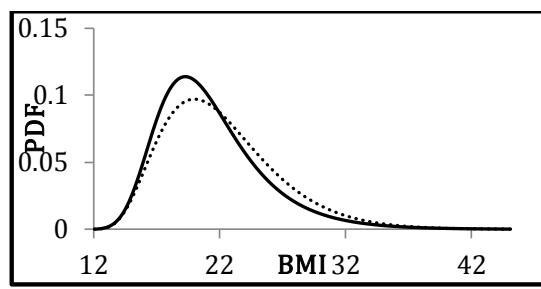

Age Group 45-49

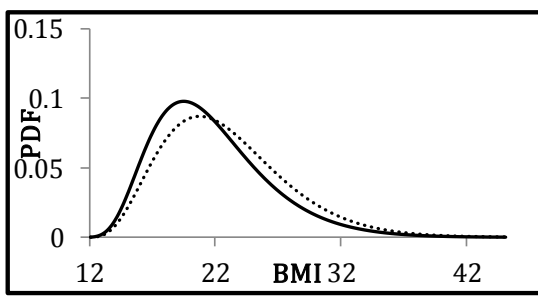

Age Group 40-44

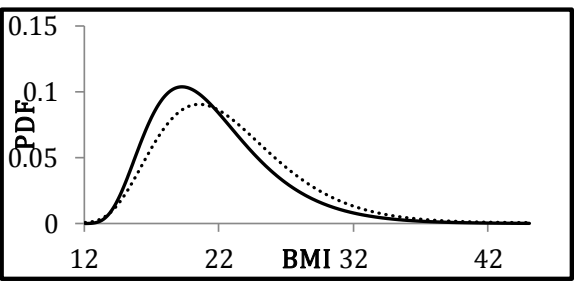

Age Group 15-49(Total)

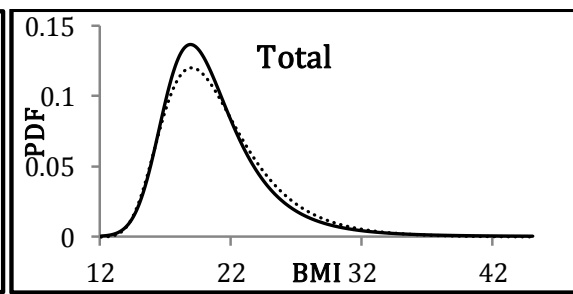

Figure 1: Change in distribution of BMI from NFHS-2 to NFHS-3 among different age groups of women using best fitted distributions (bold line: NFHS-2; dotted line: NFHS-3)

Table 5: Best fitted probability distributions to $B M I$

\begin{tabular}{|c|c|c|c|c|}
\hline $\begin{array}{l}\text { Sr. } \\
\text { No. }\end{array}$ & $B C$ & Survey & Distribution & Estimated Parameters \\
\hline \multicolumn{5}{|c|}{ Age-group } \\
\hline \multirow{2}{*}{1} & \multirow{2}{*}{ 15-19 } & NFHS-2 & Burr (4P) & $k=1.9908, \alpha=4.688, \beta=8.7118, \gamma=11.85$ \\
\hline & & NFHS-3 & Burr (4P) & $k=1.4819, \alpha=4.6931, \beta=8.5143, \gamma=11.341$ \\
\hline \multirow{2}{*}{2} & \multirow{2}{*}{$20-24$} & NFHS-2 & Burr (4P) & $k=1.3073, \alpha=4.8995, \beta=8.1063 \gamma=11.559$ \\
\hline & & NFHS-3 & Johnson SU & $=-1.7233,=1.9946, \lambda=3.8559, \xi=15.729$ \\
\hline \multirow{2}{*}{3} & \multirow{2}{*}{$25-29$} & NFHS-2 & Dagum (4P) & $k=5.2789, \alpha=14.128, \beta=28.396, \gamma=-13.116$ \\
\hline & & NFHS-3 & GEV & $k=0.01116, \sigma=2.9148, \mu=19.1$ \\
\hline \multirow{2}{*}{4} & \multirow{2}{*}{$30-34$} & NFHS-2 & GEV & $k=0.03425, \sigma=2.9243, \mu=19.021$ \\
\hline & & NFHS-3 & Fatigue Life(3P) & $\alpha=0.34504, \beta=11.29, \gamma=9.5136$ \\
\hline \multirow{2}{*}{5} & \multirow{2}{*}{$35-39$} & NFHS-2 & GEV & $k=0.02407, \sigma=3.2372, \mu=19.357$ \\
\hline & & NFHS-3 & Johnson SB & $=3.4372,=2.0883, \lambda=66.896, \xi=10.596$ \\
\hline \multirow{2}{*}{6} & \multirow{2}{*}{$40-44$} & NFHS-2 & Johnson SB & $=4.2894,=2.1029, \lambda=85.449, \xi=10.913$ \\
\hline & & NFHS-3 & Johnson SB & $=3.3016,=2.1798, \lambda=68.159, \xi=9.6363$ \\
\hline \multirow{2}{*}{7} & \multirow{2}{*}{$45-49$} & NFHS-2 & Johnson SB & $=3.7846,=2.0931, \lambda=75.194, \xi=10.368$ \\
\hline & & NFHS-3 & Beta & $\alpha_{1}=4.4617, \alpha_{2}=42.786, \mathrm{a}=11.875, \mathrm{~b}=127.82$ \\
\hline \multicolumn{5}{|c|}{ Education } \\
\hline \multirow[t]{2}{*}{8} & \multirow{2}{*}{$\begin{array}{c}\text { No } \\
\text { Education }\end{array}$} & NFHS-2 & Dagum (4P) & $k=2.3745, \alpha=9.4292, \beta=16.458, \gamma=0.69208$ \\
\hline & & NFHS-3 & Dagum (3P) & $k=3.7621, \alpha=8.253, \beta=16.05$ \\
\hline 9 & Primary & NFHS-2 & $\operatorname{Dagum}(3 \mathrm{P})$ & $k=3.6341, \alpha=7.9927, \beta=16.331$ \\
\hline
\end{tabular}




\begin{tabular}{|c|c|c|c|c|}
\hline \multirow{3}{*}{10} & & NFHS-3 & GEV & $k=0.03891, \sigma=2.9594, \mu=19.062$ \\
\hline & \multirow{2}{*}{ Secondary } & NFHS-2 & GEV & $k=0.01641, \sigma=3.2376, \mu=19.701$ \\
\hline & & NFHS-3 & GEV & $k=0.03358, \sigma=3.1811, \mu=19.326$ \\
\hline \multirow[t]{2}{*}{11} & \multirow[t]{2}{*}{ Higher } & NFHS-2 & $\begin{array}{l}\text { General Gamma } \\
(4 \mathrm{P})\end{array}$ & $k=0.80816, \alpha=12.035, \beta=0.56067 \gamma=10.603$ \\
\hline & & NFHS-3 & Gamma (3P) & $\alpha=6.3654, \beta=1.768, \gamma=11.5$ \\
\hline \multicolumn{5}{|c|}{ Standard of living } \\
\hline \multirow{2}{*}{12} & \multirow{2}{*}{ Low } & NFHS-2 & Burr (4P) & $k=1.3802, \alpha=4.9739, \beta=8.4372 \gamma=11.006$ \\
\hline & & NFHS-3 & Dagum (4P) & $k=3.4888, \alpha=24.151, \beta=41.874 \gamma=-25.829$ \\
\hline \multirow{2}{*}{13} & \multirow{2}{*}{ Medium } & NFHS-2 & Johnson SU & $=-2.3212,=2.1597, \lambda=3.9824, \xi=14.585$ \\
\hline & & NFHS-3 & GEV & $k=-0.02383, \sigma=2.6019, \mu=18.653$ \\
\hline \multirow[t]{2}{*}{14} & \multirow{2}{*}{ High } & NFHS-2 & $\begin{array}{l}\text { General Gamma } \\
\text { (4P) }\end{array}$ & $k=0.78852, \alpha=10.625, \beta=0.57405 \gamma=11.249$ \\
\hline & & NFHS-3 & Johnson SB & $=4.1745,=2.2288, \lambda=81.803, \xi=10.604$ \\
\hline \multicolumn{5}{|c|}{ Residence } \\
\hline \multirow{2}{*}{15} & & NFHS-2 & Johnson SB & $=3.5968,=2.056, \lambda=70.71, \xi=10.604$ \\
\hline & Rural & NFHS-3 & Dagum(3P) & $k=2.5567, \alpha=9.5454, \beta=17.086$ \\
\hline 16 & Urhan & NFHS-2 & Dagum (4P) & $k=1.2426, \alpha=4.1338, \beta=8.362 \gamma=11.765$ \\
\hline 10 & Urban & NFHS-3 & Johnson SB & $=4.4382,=2.3263, \lambda=89.44, \xi=10.083$ \\
\hline Relig & & & & \\
\hline & & NFHS-2 & $\operatorname{Dagum}(3 \mathrm{P})$ & $k=3.5322, \alpha=8.2031, \beta=16.385$ \\
\hline 17 & Hindu & NFHS-3 & GEV & $k=0.04838, \sigma=3.0192, \mu=19.952$ \\
\hline 18 & & NFHS-2 & $\operatorname{Dagum}(3 \mathrm{P})$ & $k=3.202, \alpha=7.8586, \beta=16.79$ \\
\hline 18 & Musıim & NFHS-3 & Pearson 6 (4P) & $\alpha_{1}=28.33, \alpha_{2}=11.045, \beta=4.0709, \gamma=9.7351$ \\
\hline & & NFHS-2 & Dagum(3P) & $k=2.0483, \alpha=9.6398, \beta=18.892$ \\
\hline 19 & Christian & NFHS-3 & Burr (4P) & $k=0.79944, \alpha=8.0183, \beta=12.125, \gamma=8.2677$ \\
\hline 20 & Sikh & NFHS-2 & Johnson SB & $=2.7399,=1.8376, \lambda=56.635, \xi=11.838$ \\
\hline 20 & Sikn & NFHS-3 & Beta & $\alpha_{1}=4.318, \alpha_{2}=346.64, \mathrm{a}=12.641, \mathrm{~b}=868.86$ \\
\hline 21 & Other & NFHS-2 & Burr & $k=0.65155, \alpha=13.297, \beta=20.048$ \\
\hline 21 & Uther & NFHS-3 & Burr (4P) & $k=1.8415, \alpha=3.552, \beta=10.645, \gamma=12.36$ \\
\hline Caste & & & & \\
\hline 22 & $\mathrm{SC}$ & NFHS-2 & Dagum(3P) & $k=2.7615, \alpha=9.2195, \beta=16.814$ \\
\hline 22 & $\mathrm{SC}$ & NFHS-3 & GEV & $k=0.04855, \sigma=2.8147, \mu=18.585$ \\
\hline & & NFHS-2 & Burr (4P) & $k=1.4562, \alpha=4.9002, \beta=9.2934 \gamma=11.199$ \\
\hline 23 & ST & NFHS-3 & Burr (4P) & $k=1.6694, \alpha=4.498, \beta=9.7852, \gamma=11.54$ \\
\hline 24 & $\mathrm{OBC}$ & NFHS-2 & Johnson SU & $=-2.1535,=2.0391, \lambda=3.8526, \xi=14.851$ \\
\hline 27 & SDC & NFHS-3 & GEV & $k=0.04924, \sigma=2.9525, \mu=18.89$ \\
\hline 25 & Other & NFHS-2 & GEV & $k=0.03162, \sigma=3.1769, \mu=19.447$ \\
\hline 25 & Uther & NFHS-3 & Johnson SB & $=3.4178,=1.878, \lambda=65.837, \xi=11.797$ \\
\hline Mari & status & & & \\
\hline 26 & Married & NFHS-2 & Dagum(3P) & $k=3.428, \alpha=8.1146, \beta=16.575$ \\
\hline 20 & Mlarried & NFHS-3 & Johnson SB & $=3.9161,=1.9673, \lambda=74.525, \xi=11.752$ \\
\hline 27 & Other & NFHS-2 & Johnson SU & $=-2.9816,=2.2275, \lambda=3.6218, \xi=13.299$ \\
\hline 21 & Utner & NFHS-3 & Burr (4P) & $k=1.315, \alpha=4.2024, \beta=8.6392, \gamma=11.571$ \\
\hline Zone & & & & \\
\hline & & NFHS-2 & GEV & $k=0.03162, \sigma=3.1769, \mu=19.447$ \\
\hline 28 & North & NFHS-3 & GEV & $k=0.04371, \sigma=3.2033, \mu=19.438$ \\
\hline
\end{tabular}




\begin{tabular}{|c|c|c|c|c|}
\hline \multirow{2}{*}{29} & \multirow{2}{*}{ Central } & NFHS-2 & Burr (4P) & $k=0.9137, \alpha=5.7572, \beta=8.596 \gamma=10.646$ \\
\hline & & NFHS-3 & $\operatorname{Dagum}(3 \mathrm{P})$ & $k=6.8874, \alpha=7.5651, \beta=14.563$ \\
\hline \multirow{2}{*}{30} & \multirow{2}{*}{ East } & NFHS-2 & Burr (4P) & $k=0.9137, \alpha=5.7572, \beta=8.596 \gamma=10.646$ \\
\hline & & NFHS-3 & GEV & $k=0.06089, \sigma=2.7207, \mu=18.527$ \\
\hline \multirow{2}{*}{31} & \multirow{2}{*}{$\begin{array}{c}\text { North- } \\
\text { east }\end{array}$} & NFHS-2 & Dagum (4P) & $k=0.76817, \alpha=6.7411, \beta=10.158 \gamma=10.812$ \\
\hline & & NFHS-3 & Burr (4P) & $k=1.3479, \alpha=5.0223, \beta=10.218, \gamma=9.7551$ \\
\hline \multirow{2}{*}{32} & \multirow{2}{*}{ West } & NFHS-2 & IG (3P) & $\lambda=65.226, \mu=10.819, \gamma=10.165$ \\
\hline & & NFHS-3 & $\mathrm{IG}(3 \mathrm{P})$ & $\lambda=72.461, \mu=11.31, \gamma=9.7551$ \\
\hline \multirow{2}{*}{33} & \multirow{2}{*}{ South } & NFHS-2 & Johnson SB & $=5.04497,=2.2418, \lambda=97.21, \xi=11.014$ \\
\hline & & NFHS-3 & Johnson SB & $=3.0867,=1.8248, \lambda=61.081, \xi=11.393$ \\
\hline \multirow{2}{*}{34} & \multirow{2}{*}{ Total } & NFHS-2 & Dagum (3P) & $k=3.3902, \alpha=8.1104, \beta=16.588$ \\
\hline & & NFHS-3 & GEV & $k=0.03899, \sigma=3.0607, \mu=19.125$ \\
\hline
\end{tabular}

GEV: General extreme value

IG: Inverse Gaussian

\subsubsection{Change in distribution of BMI by using partial sums on percentiles}

In this section, we suggest using the partial sum plot to demonstrate changes in the distribution of BMI. Changes in distribution of BMI can be shown through the changes in partial sums of absolute difference between percentiles of $B M I$ based on NFHS-2 and NFHS-3. We can compare these values of partial sums for different $B C$ with the partial sums of total population or population under other $B C$. The partial sums for some $B C$ and for some selected percentiles are reported in Table 6 and shown in Figure 2 (A, B, C and D). The numerical values from the Table 6 and Figure 2 more clearly indicate the magnitude of changes in the distribution of BMI of women from NFHS-2 to NFHS-3.

Table 6: Partial sum of absolute differences in the percentiles of distribution of $B M I$ in NFHS-2 and NFHS-3 for selected BC

\begin{tabular}{|c|c|c|c|c|c|c|}
\hline \multirow{3}{*}{$\begin{array}{c}\text { Background } \\
\text { Characteristic }(\boldsymbol{B C})\end{array}$} & \multicolumn{5}{|c|}{ Partial sum up to Percentiles } \\
\cline { 2 - 7 } & $\mathbf{1 0}^{\text {th }}$ & $\mathbf{2 0}^{\text {th }}$ & $\mathbf{5 0}^{\text {th }}$ & $\mathbf{8 0}^{\text {th }}$ & $\mathbf{9 8}^{\text {th }}$ \\
\hline \multirow{3}{*}{ Residence } & Rural & 0.1 & 0.35 & 1.05 & 2.42 & 7.77 \\
\cline { 2 - 7 } & Urban & 1.03 & 2.36 & 6.34 & 8.21 & 10.11 \\
\cline { 2 - 7 } & Total & 0.12 & 0.4 & 0.79 & 4.42 & 10.87 \\
\hline \multirow{4}{*}{ Age-group } & $15-19$ & 2.56 & 4.97 & 11.35 & 17.29 & 19.34 \\
\cline { 2 - 7 } & $20-24$ & 0.49 & 0.93 & 2.06 & 3.76 & 10.84 \\
\cline { 2 - 7 } & $25-29$ & 0.22 & 0.39 & 2.95 & 11.05 & 21.04 \\
\cline { 2 - 7 } & $30-34$ & 0.27 & 0.37 & 2.5 & 11.32 & 19.05 \\
\cline { 2 - 7 } & $35-39$ & 0.58 & 1.12 & 5.4 & 16.45 & 25.24 \\
\cline { 2 - 7 } & $40-44$ & 0.83 & 2.18 & 9.83 & 24.97 & 36.45 \\
\cline { 2 - 7 } & $45-49$ & 1.93 & 4.08 & 12.45 & 27.54 & 36.32 \\
\hline \multirow{4}{*}{ Education } & No Education & 0.08 & 0.16 & 0.39 & 3.7 & 12.33 \\
\cline { 2 - 7 } & Primary & 0.12 & 0.2 & 0.69 & 3.42 & 8.73 \\
\cline { 2 - 7 } & Secondary & 1.5 & 3.58 & 11.25 & 18.27 & 19.53 \\
\cline { 2 - 7 } & Higher & 1.91 & 4.22 & 10.86 & 15.34 & 18.81 \\
\hline
\end{tabular}




\begin{tabular}{|c|c|c|c|c|c|c|}
\hline \multirow{3}{*}{$\begin{array}{c}\text { Standard of } \\
\text { living }\end{array}$} & Low & 0.2 & 0.57 & 2.37 & 4.64 & 6.18 \\
\cline { 2 - 7 } & Medium & 1.08 & 2.55 & 7.69 & 14.32 & 18.84 \\
\cline { 2 - 7 } & High & 2.85 & 6.44 & 19.32 & 32.24 & 38.06 \\
\hline
\end{tabular}

(A)

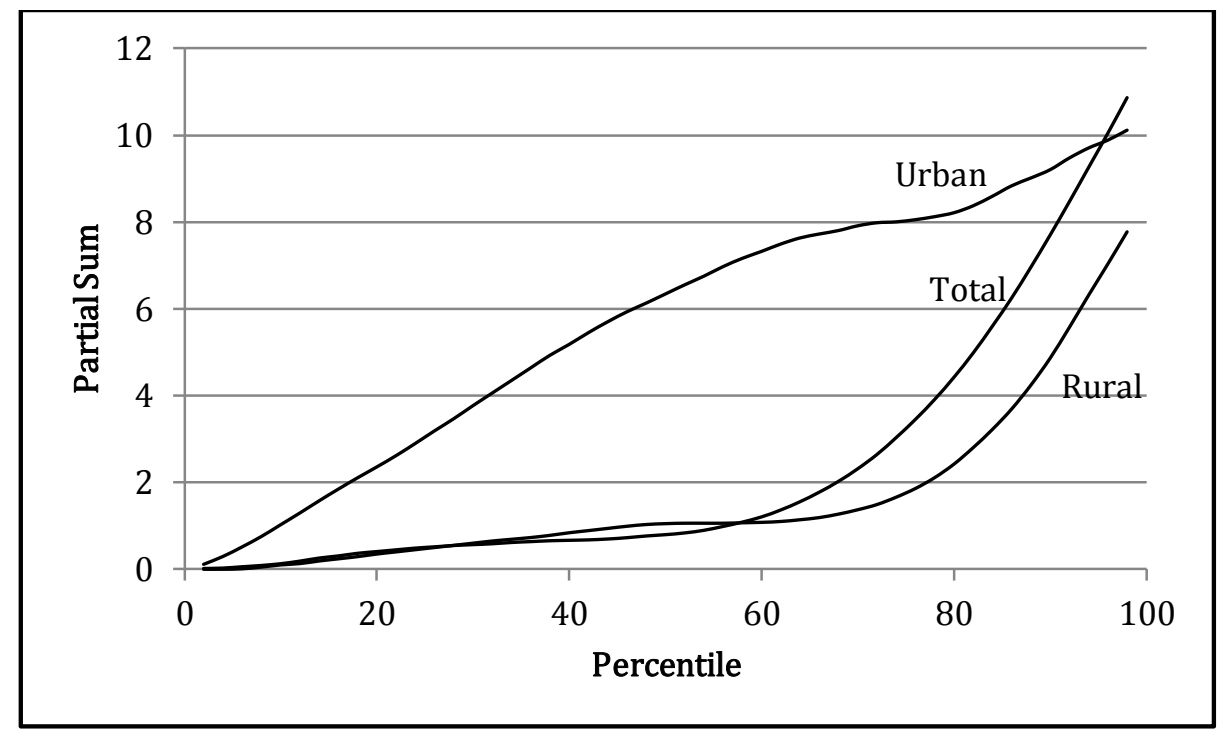

(B)

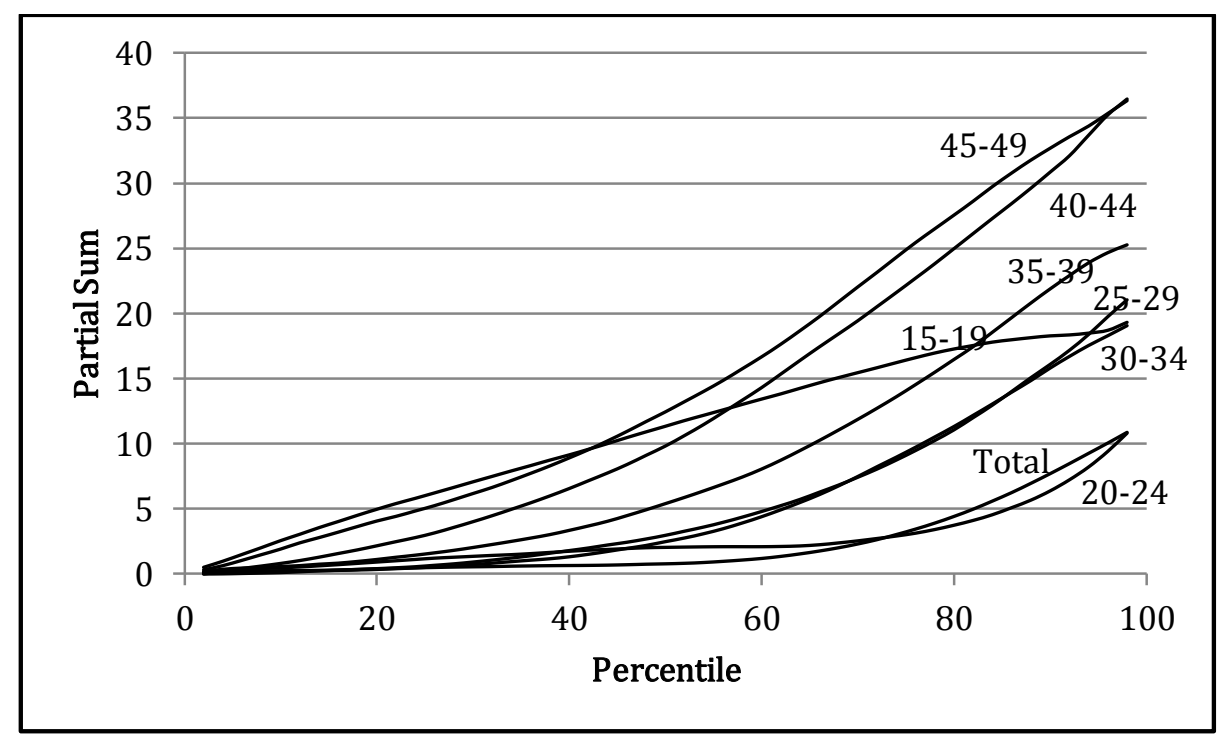


(C)

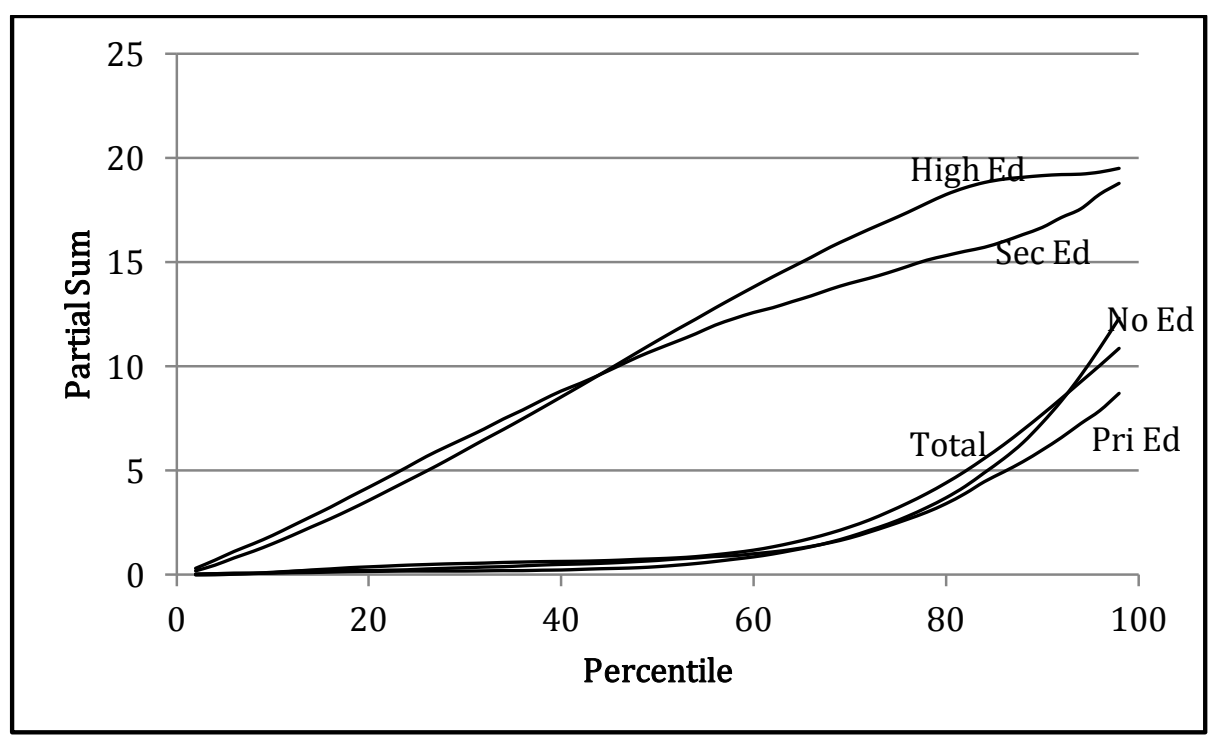

(D)

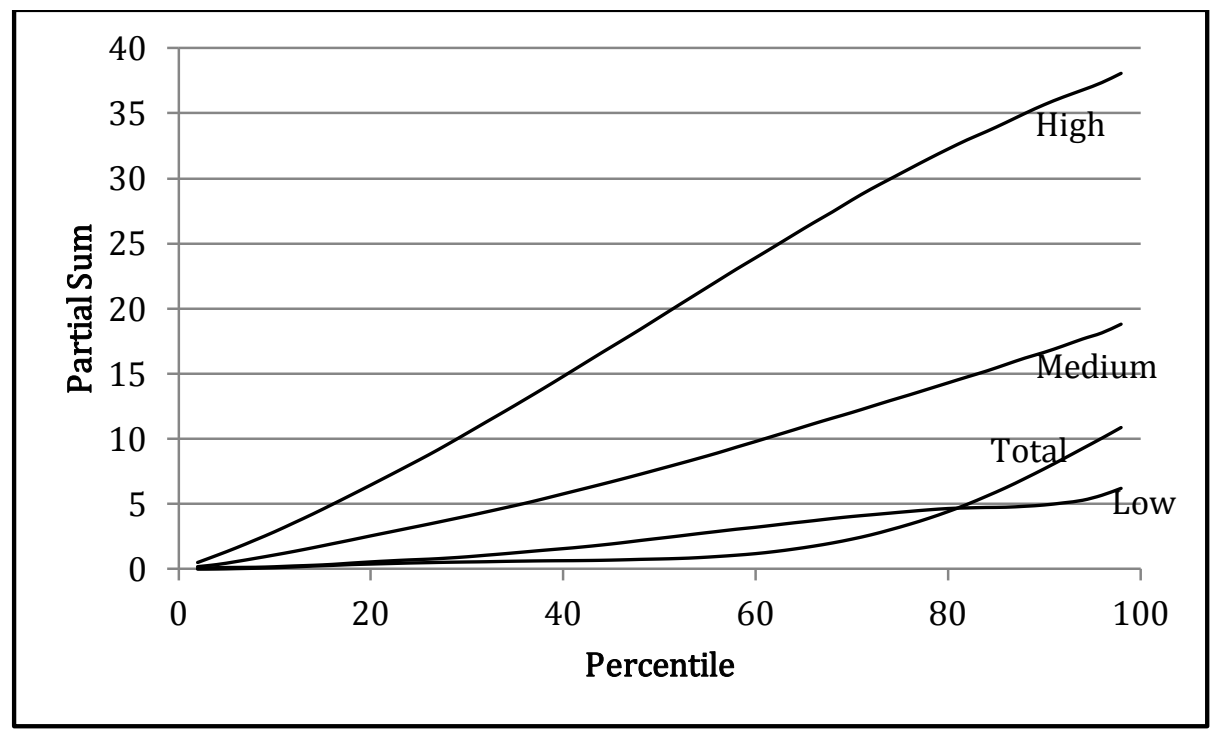

Figure 2: Change in distribution using partial sum according to (A) type of residence (B) age-group (C) education and (D) standard of living 


\subsubsection{Change in distribution of $B M I$ using test of equality of percentiles}

Student's t-test and analysis of variance are frequently used to test the hypothesis that two or more distributions have same means. However, many random variables such as $B M I$ having skewed distributions that are not readily transformed to symmetry, rendering the distributional assumptions that underlie use of these methods inappropriately. The non-parametric tests such as Wilcoxon, Kolmogorov-Smirnov, and median tests are used to test for differences in distributions (Siegel \&Castellan, 1988). These tests are not designed to pin-point where the distributions are unequal or simultaneously test for differences in more than one distribution parameter. The t-test and variance-test are powerful for detecting differences in location and scale respectively. The t-test and variancetest have scope to test one parameter only. In these circumstances, it may be of greater interest to compare the distributions in terms of their percentiles rather than their means or an overall test of equivalence. A percentile profile is defined as a set of one or more percentiles. It may be more informative to compare two or more distributions by testing the hypothesis that their profiles of judiciously selected percentiles are equal (Zhang et al. 2014).

Zhang et al. (2014) had first described the procedure as a generalization of the median test. In median test, we test the equality of the $50^{\text {th }}$ percentile. Instead of testing the equality of only one percentile, the method is extended to simultaneously test multiple percentiles. The method is as follows:

Let $Y$ denote a continuous random variable (in our case $Y$ is $B M I$ ) and let $Q_{1}, Q_{2}$, $\ldots, Q_{m}$ denote a set of $m$ percentiles that in some sense characterize the distribution of $B M I$ across its range. Further let $y_{1}, y_{2}, \ldots, y_{n}$ represent a random sample of observations and let $q_{1}, q_{2}, \ldots, q_{m}$ represent the usual sample estimates of $Q_{1}, Q_{2}, \ldots, Q_{m}$ respectively. Suppose random samples are available from each of $k$ populations with percentiles $Q_{h 1}, Q_{h 2}, \ldots, Q_{h m}, h=1,2, \ldots, k$. There is interest in testing the hypothesis that the percentile profiles are identical across the $k$ populations; that is to test $\mathrm{H}_{0}: Q_{1 i}=Q_{2 i}=\ldots=Q_{k i} i=1,2, \ldots, m$. The complete process of testing this hypothesis as an extension of median test was discussed in Zhang et al. (2014). We have conducted these tests for $k=2$ (as we have two distributions of $B M I$ one each for NFHS-2 and NFHS-3) and $p=5$ $\left(5^{\text {th }}, 25^{\text {th }}, 50^{\text {th }}, 75^{\text {th }}\right.$ and $95^{\text {th }}$ percentiles). The $p$-values of test of equality of percentiles of $B M I$ for five percentiles and for five combined percentiles are reported in the Table 7. 
Table 7: Test of equality of percentiles of BMI from NFHS-2 to NFHS-3

\begin{tabular}{|c|c|c|c|c|c|c|}
\hline \multirow{2}{*}{$B C$} & \multicolumn{5}{|c|}{ p-values of test of equality for percentiles } & \multirow{2}{*}{$\begin{array}{l}\text { Combined } \\
\text { percentiles }\end{array}$} \\
\hline & $\mathbf{P}_{5}$ & $\mathbf{P}_{25}$ & $\mathbf{P}_{50}$ & $\mathbf{P}_{75}$ & $\mathbf{P}_{95}$ & \\
\hline \multicolumn{7}{|l|}{ Age-group } \\
\hline $15-19$ & $<0.001$ & $<0.001$ & $<0.001$ & $<0.002$ & $<0.001$ & $<0.001$ \\
\hline $20-24$ & 0.06 & 0.47 & $<0.001$ & $<0.001$ & $<0.001$ & $<0.001$ \\
\hline $25-29$ & 0.3535 & $<0.001$ & $<0.001$ & $<0.001$ & $<0.001$ & $<0.001$ \\
\hline $30-34$ & 0.18 & $<0.001$ & $<0.001$ & $<0.001$ & $<0.001$ & $<0.001$ \\
\hline $35-39$ & 0.0010 & $<0.001$ & $<0.001$ & $<0.001$ & $<0.001$ & $<0.001$ \\
\hline $40-44$ & $<0.001$ & $<0.001$ & $<0.001$ & $<0.001$ & $<0.001$ & $<0.001$ \\
\hline $45-49$ & $<0.001$ & $<0.001$ & $<0.001$ & $<0.001$ & $<0.001$ & $<0.001$ \\
\hline \multicolumn{7}{|l|}{ Residence } \\
\hline Rural & 0.93 & 0.33 & $<0.001$ & $<0.001$ & $<0.001$ & $<0.001$ \\
\hline Urban & $<0.001$ & $<0.001$ & $<0.001$ & 0.02 & 0.94 & $<0.001$ \\
\hline \multicolumn{7}{|l|}{ Education } \\
\hline No Education & 0.29 & .0016 & $<0.001$ & $<0.001$ & $<0.001$ & $<0.001$ \\
\hline Primary & 0.27 & .0049 & $<0.001$ & $<0.001$ & $<0.001$ & $<0.001$ \\
\hline Secondary & $<0.001$ & $<0.001$ & $<0.001$ & $<0.001$ & $<0.001$ & $<0.001$ \\
\hline Higher & $<0.001$ & $<0.001$ & $<0.001$ & $<0.001$ & $<0.001$ & $<0.001$ \\
\hline \multicolumn{7}{|c|}{ Standard of living } \\
\hline Low & 0.49 & 0.05 & 0.0025 & 0.51 & $<0.001$ & $<0.001$ \\
\hline Medium & $<0.001$ & $<0.001$ & $<0.001$ & $<0.001$ & 0.02 & $<0.001$ \\
\hline High & $<0.001$ & $<0.001$ & $<0.001$ & $<0.001$ & $<0.001$ & $<0.001$ \\
\hline \multicolumn{7}{|l|}{ Religion } \\
\hline Hindu & 0.08 & $<0.001$ & $<0.001$ & $<0.001$ & $<0.001$ & $<0.001$ \\
\hline Muslim & 0.82 & 0.34 & 0.0031 & $<0.001$ & $<0.001$ & $<0.001$ \\
\hline Christian & $<0.001$ & $<0.001$ & 0.08 & 0.77 & 0.59 & 0.0019 \\
\hline Sikh & 0.79 & 0.54 & 0.55 & .06 & 0.35 & 0.36 \\
\hline Other & 0.25 & $<0.001$ & 0.1008 & 0.38 & 0.70 & $<0.001$ \\
\hline \multicolumn{7}{|l|}{ Caste } \\
\hline $\mathrm{SC}$ & 0.35 & 0.04 & $<0.001$ & $<0.001$ & $<0.001$ & $<0.001$ \\
\hline ST & 0.0018 & $<0.001$ & $<0.001$ & $<0.001$ & $<0.001$ & $<0.001$ \\
\hline $\mathrm{OBC}$ & 0.19 & 0.20 & $<0.001$ & $<0.001$ & $<0.001$ & $<0.001$ \\
\hline Other & 0.92 & 0.0050 & $<0.001$ & $<0.001$ & $<0.001$ & $<0.001$ \\
\hline \multicolumn{7}{|l|}{ Marital status } \\
\hline Married & $<0.001$ & $<0.001$ & $<0.001$ & $<0.001$ & $<0.001$ & $<0.001$ \\
\hline Other & 0.64 & 0.08 & $<0.001$ & $<0.001$ & $<0.001$ & $<0.001$ \\
\hline \multicolumn{7}{|l|}{ Zone } \\
\hline North & 0.0171 & 0.06 & 0.05 & $<0.001$ & 0.0124 & $<0.001$ \\
\hline Central & 0.01 & 0.31 & 0.03 & $<0.001$ & $<0.001$ & $<0.001$ \\
\hline East & 0.10 & 0.0022 & $<0.001$ & $<0.001$ & $<0.001$ & $<0.001$ \\
\hline North-East & 0.09 & 0.01 & $<0.001$ & $<0.001$ & $<0.001$ & $<0.001$ \\
\hline West & 0.99 & 0.33 & 0.55 & 0.07 & 0.49 & 0.53 \\
\hline South & 0.94 & $<0.001$ & $<0.001$ & $<0.001$ & $<0.001$ & $<0.001$ \\
\hline
\end{tabular}




\begin{tabular}{|c|c|c|c|c|c|c|}
\hline Total & 0.99 & $<0.001$ & $<0.001$ & $<0.001$ & $<0.001$ & $<0.001$ \\
\hline
\end{tabular}

\section{Results and Discussion}

The weighted and un-weighted prevalence according to different $B C$ of Indian women during NFHS-2 and NFHS-3 are presented in Table 1. For all Indian women, both the prevalence rates of UW remained almost same and obesity (OW/OB) increased from NFHS-2 to NFHS-3. Under both the surveys, for all women the value of $\mathrm{R}_{1}$ increased from 1.16 to 1.31 , which shows that more than $50 \%$ of Indian women were under malnutrition (UW/OW/OB) status under both surveys. For the age-group 15-19 years, prevalence of UW has increased from $38.6 \%$ in NFHS-2 to $46.8 \%$ in NFHS-3. Age-groups with ages above 25 years have shown decrements in prevalence of UW from NFHS-2 to NFHS-3. In all age-groups prevalence of obesity has increased from NFHS-2 to NFHS-3. Prevalence of obesity has decreased for women with secondary and higher education but total prevalence of obesity in higher education category was still high (40.8\%) in NFHS-3 as compared to $43.8 \%$ in NFHS-2. Overall it is observed that education levels affected more on prevalence of UW as well as obesity in both surveys. Under both surveys, we observed double burden of UW as well as obesity among women from the religions, Sikh $\left(\mathrm{R}_{1}=1.72, \mathrm{R}_{2}=2.56\right.$ in NFHS-3) and Christian $\left(\mathrm{R}_{1}=1.18, \mathrm{R}_{2}=1.34\right.$ in NFHS-3). High level $(46.6 \%)$ of UW is observed for ST caste category women in NFHS-3. More burdens of UW and obesity are observed for the General and other caste category in NFHS-3. In the order of caste categories as ST, SC, OBC and General and other, mean and SD of $B M I$ are observed in increasing order. This shows that deprivation of socially backward classes was continued in India till 2006. As per NFHS-3, unmarried and other women are more likely (42.8\%) to be UW than married women (33\%). Prevalence of obesity for married women was $24.4 \%$ as compared to $12.3 \%$ for unmarried and other class in NFHS-3. We observed highest burden of UW $(41.9 \%)$ in East zone, high level of obesity in South zone (29\%) and North zone (26.5\%) under NFHS-3.

Changes in descriptive statistics of $B M I$ according to $B C$ over two surveys are presented in Table 2. Mean and SD of $B M I$ of all Indian women has increased from 20.28 to 20.47 and 3.68 to 3.93 respectively. For the comparison of the tails of the distribution of $B M I$ we observed that major changes occurred at the $95^{\text {th }}$ percentile than at the $5^{\text {th }}$ percentile for many $B C$. In the group of women with high living standard, double burden of UW and obesity was continued fromNFHS-2 to NFHS-3. Mean BMI increased at high rate for married, women with ages 25-49 and women from south zone of India. Values of coefficient of skewness of the distribution of $B M I$ for all Indian women and for many $B C$ have decreased but still values were close to one or greater than one under NFHS- 
3 which shows highly positively skewed distributions of $B M I$. For the women age-group 15-19 and women with low standard of living the values of coefficient of skewness of the distribution of $B M I$ have increased and observed to be very high.

From Table 3, under synthetic cohort age-groups, we observed negative changes in the prevalence percentages for UW and NW, whereas positive changes are reported for OW and $\mathrm{OB}$ classes. High positive changes are reported for women from obese class. We noticed from Table 4 that an annual gain in mean $B M I$ was highest $(0.82 \%)$ for the age-group 22-28 under NFHS-2 with its cohort group 2935 under $N F H S$-3. Also, as expected, annual gains in mean $B M I$ were higher for cohort groups than same age groups.

Best fitted probability distributions for $B M I$ of women under different $B C$ were decided based on p-values of goodness of fit tests and ranks given by Easy-Fit software. These best fitted distributions are reported in Table 5. Changes in the distribution of $B M I$ under different age-groups using best fitted distributions and their estimated parameters are demonstrated in Figure 1. Due to lack of space similar figures for other $B C$ are not presented in this manuscript.

From Table 6 and Figure 2, we clearly observed the magnitude of changes in distribution of $B M I$ in terms of percentiles of distribution. For urban women, women from older age-groups, women with secondary/higher education and women with high standard of living almost uniform changes in all percentiles are observed, whereas for other $B C$ major changes are observed at higher percentiles of BMI. From Table 7 we notice that, tests of equality of percentiles of $B M I$ showed significant difference in percentile of BMI for higher percentiles of NFHS-2 and NFHS-3 for many BC. Test of equality of percentiles can be accepted for all and combined percentiles for women from Sikh religion and women from West zone of India.

Our results suggest that, rapid increments are observed in mean and higher percentiles of $B M I$ among married women, women in the higher age-groups, and women from south zone. This study shows a persistent problem of UW in India, with $35.2 \%$ and $35.6 \%$ of women population being UW in NFHS-2 and NFHS-3 respectively; at the same time the percentage of women who are overweight and obese has increased from $18.4 \%$ to $21.2 \%$. This pattern of persisting problems of under nutrition along with a simultaneous rise in obesity was also seen in most of the low to middle income countries (Razaket al., 2013). Using a synthetic cohort approach we have quantified age-specific longitudinal change in BMI among Indian women during from NFHS-2 to NFHS-3. The positive changes in prevalence percentages according to $B M I$ categories for all synthetic cohort age- 
groups are observed for $\mathrm{OW} / \mathrm{OB}$ categories while negative changes for all synthetic cohort age-groups are observed for UW and NW categories of BMI. This shows the impact of ageing on weight gain among Indian women.

Concerning the prevalence of UW and obesity(OW/OB) there is urgent need to make more awareness of consequences of underweight and obesity among highly educated women and women from high standard of living. To overcome the problem of UW, the Indian government has passed the National Food Security Act, 2013 in September2013. As per the National Food Security Act 2013, all eligible households are entitled to get food grains ( $5 \mathrm{~kg}$ per person per month) at the subsidized prices. This Act will make access for sufficient and nutritious food for $75 \%$ of rural and $50 \%$ of urban population of India including all poor. There are some more provisions for advancing food security as mentioned in the National Food Security Act 2013, which may also be helpful to improve the nutritional status of Indian women. These provisions include access to (a) safe and adequate drinking water and sanitation; (b) health care;(c) nutritional, health, and education support to adolescents; and (d) adequate pensions for senior citizens, persons with disability, and single women (National Food Security Act, 2013). More awareness programs to control obesity among Indian women are needed. Government may think of providing some incentives to employees for consistently maintaining their $B M I$ under NW category.

\section{Conclusion}

In all age-groups except 15-19 years, the distribution $B M I$ shifted to the right and become more right skewed and extent of shift is greatest for the older age-groups. Women with low standard of living the prevalence of underweight had increased from $46.9 \%$ to $49.7 \%$ during the period NFHS-2 to NFHS-3. Also, prevalence of obesity (OW/OB) among all women increased from $18.4 \%$ to $21.2 \%$. For many $B C$ major changes in higher percentiles of distribution of $B M I$ are observed from $N F H S-2$ to NFHS-3. This shows the right shift of the distribution of BMI. As per NFHS-3, problem of obesity was still serious or started becoming serious among urban women, rich and highly educated women, women from south and north zone, women belonging to general and other caste category, and among married women.

The major limitation of this study is that, as the respondents are not same from NFHS-2 and NFHS-3, hence carrying out longitudinal study to demonstrate change in distribution of BMI based on synthetic cohorts may not be proper approach. However, we have used this approach because sample sizes are very large in both nationwide surveys, so the distribution of BMI was properly reflected. An advantage of this study is that the best fitted probability 
distributions can be used to plot cumulative distribution function of $B M I$ under different $B C$. Also, in future, researchers can use these fitted probability distributions to study longitudinal changes in the distribution of $B M I$ of Indian women.

\section{Acknowledgements}

Authors are thankful to both reviewers for giving their comments and suggestions useful for improving the presentation of manuscript.

\section{References}

1. Easy Fit software available at http://www.mathwave.com/downloads

2. National Food Security Act, 2013. (2013). Registered No. DL(N)04/0007/2003-13. Published by the Ministry of Law and Justice (Legislative Department) Government of India.

3. Flegal, K. M., Carroll, M. D., Ogden, C. L. and Curtin L. R., (2010). Prevalence and trends in obesity among US adults, 1999-2008. JAMA,303, pp. 235-241. DOI:10.1001/jama.2009.2014

4. Hedley, A. A., Ogden, C. L., Johnson, C. L., Carroll, M. D., Curtin L. R., and Flegal, K. M.,(2004). Prevalence of overweight and obesity among US children, adolescents, and adults, 1999-2002. JAMA, 291, pp.2847-2850. DOI: $10.1001 /$ jama291.23.2847

5. Ljungvall, A., and Zimmerman, F. J., (2012). Bigger bodies: long-term trends and disparities in obesity and body-mass index among U.S. adults 1960-2008. Soc Sci Med, 75 pp. 109-119. DOI: 10.1016/j.Socscimed.2012.03.003

6. Lean, M.E.J., Katsarou, C., McLoone, P. and Morrison, D.S., (2013). Changes in BMI and waist circumference in Scottish adults: use of repeated cross-sectional surveys to explore multiple age groups and birth-cohorts. International Journal of Obesity, 37(6), pp.800-808. DOI: 10.1038/ijo.2012.122

7. Komlos, J. and Brabec, M. (2011). The trend of BMI values of US adults by deciles. Birth cohorts 1882-1986 stratified by gender and ethnicity. Econ Hum Biol, 31, pp. 174-181. DOI: 10.1016/j.ehb.2011.03.005

8. National Family Health Survey (NFHS-2) (2000). International Institute for Population Sciences (IIPS) and Macro International, Available at http://www.measuredhs.com/pubs/pdf/FRIND2/FRIND2-VOL2.pdf. 
9. National Family Health Survey (NFHS-3) (2007). International Institute for Population Sciences (IIPS) and Macro International, Available at http://www.measuredhs.com/pubs/pdf/FRIND3/FRIND3-VOL2.pdf.

10. Ogden, C. L., Carroll, M. D., Kit, B. K., and Flegal, K. M., (2014). Prevalence of childhood and adult obesity in the United States, 2011-2012. JAMA, 311, pp. 806-814. DOI: 10.1001/jama.2014.732

11. Patil, Y. P. and Shinde, R. L. (2014): Undernutrition Among Indian Men A Study Based on NFHS-3. American journal of men's health,8(6): 492-502. DOI: $10.1177 / 1557988314525193$

12. Penman, A.D. and Johnson, W.D.,( 2006). The changing shape of the body mass index distribution curve in the population: implications for public health policy to reduce the prevalence of adult obesity. Prev Chronic Dis, 3(3), p.A74

13. Razak, F., Corsi, D.J. and Subramanian, S.V., (2013). Change in the body mass index distribution for women: analysis of surveys from 37 low-and middle-income countries. PLOS Med, 10(1), pp. e1001367. DOI: 10.1371/journal.pmed.1001367

14. Robinson, W.R., Keyes, K.M., Utz, R.L., Martin, C.L. and Yang, Y., (2013). Birth cohort effects among US-born adults born in the 1980s: foreshadowing future trends in US obesity prevalence. International journal of obesity, 37(3), pp.448-454. DOI: 10.1038/ijo.2012.66

15. Siegel, S. and Castellan N. J. Jr., (1988). Nonparametric Statistics for the Behavioural Sciences, $2^{\text {nd }}$ edn. New York: MacGraw-Hill.

16. World Health Organization (2004). Appropriate body mass index for Asian populations and its implications for policy and intervention strategies. Lancet; 363 pp. 157-163. DOI: 10.1016/S0140-6736(03)15268-3

17. Zhang, L, Beyl, R, Burton, J, Johnson C. M, Han H, Johnson W. D, (2014). A Large Sample Statistical Test for Equality of Percentile Profiles across Multiple Populations, Proceedings of the Joint Statistical Meetings of the American Statistical Association, Biometric Society and Institute of Mathematical Statistics Section on Statistics in Epidemiology, Boston 2-7 August 2014 pp. 3851-3857. 
Appendix: Probability density functions of distribution fitted for BMI

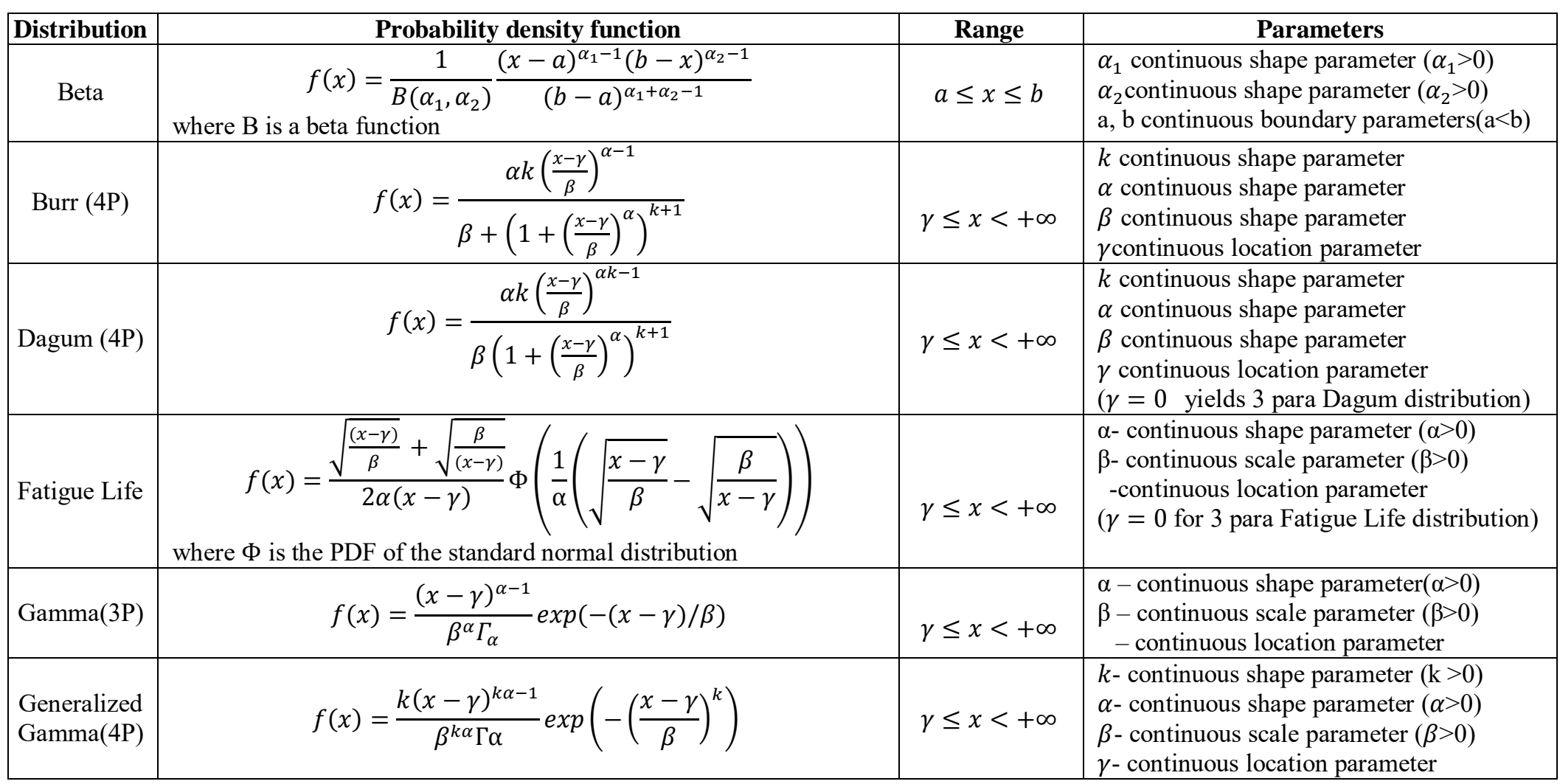




\begin{tabular}{|c|c|c|c|}
\hline Distribution & Probability density function & Range & Parameters \\
\hline $\begin{array}{l}\text { Generalized } \\
\text { Extreme } \\
\text { Value }\end{array}$ & $\begin{array}{c}f(x)= \begin{cases}\frac{1}{\sigma} \exp \left(-(1+k z)^{-1 / k}\right)(1+k z)^{-1-1 / k} & \mathrm{k} \neq 0 \\
\frac{1}{\sigma} \exp (-\mathrm{z}-\exp (-\mathrm{z})) & \mathrm{k}=0\end{cases} \\
\text { where } z=\frac{x-\mu}{\sigma}, \quad 1+\left(\frac{x-\mu}{\sigma}\right)>0 \text { for } \mathrm{k} \neq 0,\end{array}$ & $-\infty<x<+\infty$ & $\begin{array}{l}\text { k-continuous shape parameter } \\
\sigma \text {-continuous scale parameter }(\sigma>0) \\
\mu \text {-continuous location parameter }\end{array}$ \\
\hline $\begin{array}{l}\text { Gumbel } \\
\text { Max }\end{array}$ & $f(x)=\frac{1}{\sigma} \exp (-z-\exp (-z)) \quad$ where $z=\frac{x-\mu}{\sigma}$ & $-\infty<x<+\infty$ & $\begin{array}{l}\sigma \text {-continuous scale parameter }(\sigma>0) \\
\mu \text {-continuous location parameter }\end{array}$ \\
\hline Johnson SB & $f(x)=\frac{\delta}{\lambda \sqrt{2 \pi} z(1-z)} \exp \left(\left(-\frac{1}{2}\left(\gamma+\delta \ln \left(\frac{z}{(1-Z)}\right)\right)\right)^{2}\right)$ where $z=\frac{x-\xi}{\lambda}$ & $\xi \leq x \leq \xi+\lambda$ & $\begin{array}{l}\text {-continuous shape parameter } \\
\text { continuous shape parameter }(>0) \\
\lambda \text { - continuous scale parameter }(\lambda>0) \\
\xi \text { - continuous location parameter }\end{array}$ \\
\hline $\begin{array}{l}\text { Lognormal } \\
\quad(3 \mathrm{P})\end{array}$ & $f(x)=\frac{\exp \left(-\frac{1}{2}\left(\frac{\ln (x-\gamma)-\mu)}{\sigma}\right)^{2}\right)}{(x-\gamma) \sigma \sqrt{2 \pi}}$ & $\gamma \leq x<+\infty$ & $\begin{array}{l}\sigma \text {-continuous parameter }(\sigma>0) \\
\mu \text {-continuous parameter } \\
\text {-continuous location parameter }\end{array}$ \\
\hline $\begin{array}{l}\text { Pearson } \\
\text { Type } 6 \\
(4 \mathrm{P})\end{array}$ & $f(x)=\frac{((x-\gamma) / \beta)^{\alpha_{1}-1}}{\beta B\left(\alpha_{1}, \alpha_{2}\right)(1+x / \beta)^{\alpha_{1}+\alpha_{2}}}$ & $\gamma \leq x<+\infty$ & $\begin{array}{l}\alpha_{1} \text {-continuous shape parameter }\left(\alpha_{1}>0\right) \\
\alpha_{2} \text {-continuous shape parameter }\left(\alpha_{2}>0\right) \\
\beta \text {-continuous scale parameter }(\beta>0) \\
\quad \text {-continuous location parameter }\end{array}$ \\
\hline
\end{tabular}

